\title{
Homogeneous hydrogenation of saturated bicarbonate slurry to formates using multiphase catalysis
}

\author{
Christophe Rebreyend, ${ }^{a}$ Evgeny A. Pidko ${ }^{\text {a* }}$ and Georgy A. Filonenko ${ }^{\text {a* }}$
}

[a] Dr. C. Rebreyend, Prof. dr. E. A. Pidko, Dr. G. A. Filonenko

Department of Chemical Engineering

Faculty of Applied Sciences, technical University Delft

van der Maasweg 9, $2629 \mathrm{HZ}$ Delft, the Netherlands.

E-mail: G.A.Filonenko@tudelft.nl, E.A.Pidko@tudelft.n

Supporting information for this article is given via a link at the end of the document.

\begin{abstract}
Formic acid and formate salts are key intermediates along the pathways for $\mathrm{CO}_{2}$ utilization and hydrogen storage. Herein we report a highly efficient multiphase catalytic system utilizing ruthenium PNP pincer catalyst for converting supersaturated bicarbonate solutions and slurries to aqueous formate solutions up to $12 \mathrm{M}$ in molarity. The biphasic catalytic system delivers turnover frequencies up to $73000 \mathrm{~h}^{-1}$ and remains stable for up to $474^{\prime} 000$ turnovers once reaction conditions are optimized.
\end{abstract}

Among the various $\mathrm{CO}_{2}$ utilization strategies, its chemical conversion to $\mathrm{C}_{1}$ building blocks have been the focus of intense research effort over the last decades. ${ }^{[1]}$ The common utilization routes rely on $\mathrm{CO}_{2}$ reduction that can be performed electrochemically ${ }^{[2]}$ or utilize molecular hydrogen as a reducing agent. ${ }^{[3]}$ The latter can be further split into heterogeneous processes that mainly target methanol as a main product and homogeneous reduction protocols that typically produce formic acid and its salts. ${ }^{[4]}$ Although recent years have seen significant progress in base metal catalysts for $\mathrm{CO}_{2}$ hydrogenation, namely iron, ${ }^{[5]}$ cobalt ${ }^{[6]}$ and manganese, ${ }^{[7]}$ the state-of-the-art in homogeneous $\mathrm{CO}_{2}$ hydrogenation is dominated by noble metal catalysts. Figure 1 depicts several representative examples of such complexes. One of the first highly active $\mathrm{CO}_{2}$ hydrogenation catalysts reported by Nozaki and co-workers ${ }^{[4 \mathrm{q}]}$ (2, Figure 1) shows exceptional turnover frequencies (TOFs) up to $150000 \mathrm{~h}^{-}$ 1 in hydrogenating $\mathrm{CO}_{2}$ in the presence of aqueous $\mathrm{KOH} / \mathrm{THF}$ mixtures at $120-200^{\circ} \mathrm{C}$. A family of iridium catalysts reported by Himeda and co-workers $\left.{ }^{[4 a}, 4 \mathrm{~b}, 4 \mathrm{f}, 4 \mathrm{~g}, 8\right]$ with complex 3 (Figure 1) as a representative example were also noted for their $\mathrm{CO}_{2}$ hydrogenation activity pronounced in aqueous at room temperature and 1 bar of hydrogen pressure. Importantly, operation of these complexes could be switched between $\mathrm{CO}_{2}$ hydrogenation and formic acid dehydrogenation by controlling $\mathrm{pH}$ of the reaction solution. Finally, our group reported the highly active ruthenium catalyst (1, Figure 1), which was active for both $\mathrm{CO}_{2}$ hydrogenation and formate dehydrogenation albeit in organic solvents in the presence of organic bases.

From the process standpoint, homogeneous $\mathrm{CO}_{2}$ hydrogenation should fulfil several requirements to be practical. Firstly, the catalyst should be sufficiently stable to provide high, economically relevant productivity. Secondly, the catalyst should be separable form the formate product that can easily be dehydrogenated once the hydrogenation reactor is decompressed. The latter have recently been addresses by immobilizing homogeneous catalysts on solid supports ${ }^{[9]}$ and encapsulating them into porous carriers. ${ }^{[10]}$ While this approach greatly simplifies catalyst separation, it has a drawback of consistently lower activity of immobilized catalyst compared to its free molecular predecessor.

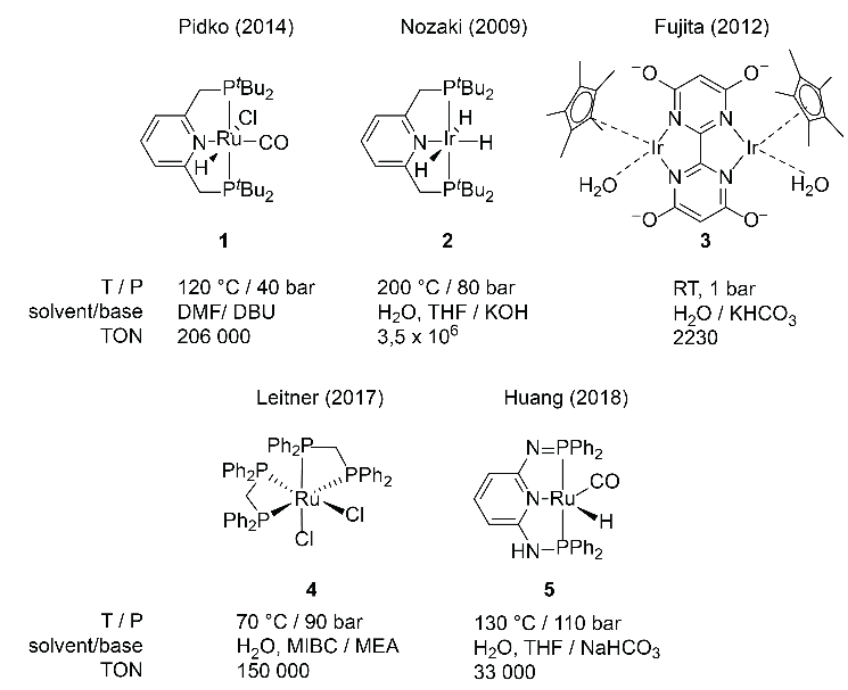

Figure 1. (a) Representative homogeneous catalysts for the hydrogenation of $\mathrm{CO}_{2}$ to formates and (b) examples of catalysts operating in biphasic systems, allowing for easy product separation.

An approach alternative to immobilization makes use of the biphasic solvent systems, ${ }^{[11]}$ in which the catalyst and reactants reside in different phases. Formate salts typically reside in aqueous phase while the organometallic catalyst species remain in the organic phase and the catalyst and product(s) separation in biphasic system can be done by simple decantation. Successful utilization of biphasic catalyst system for $\mathrm{CO}_{2}$ hydrogenation has been demonstrated with catalysts $4^{[11 \mathrm{a}]}$ and $5^{[11 \mathrm{~b}]}$ shown above and development of highly active biphasic $\mathrm{CO}_{2}$ hydrogenation is our primary goal in this work.

Herein we report the development of a highly active multiphase catalyst system based on our Ru-PNP catalyst 1 enabling hydrogenation of supersaturated aqueous bicarbonate to formate with outstanding efficiency. While complex $\mathbf{1}$ is a highly competent catalyst in fully organic media, e.g. DMF/DBU system utilized in earlier studies ${ }^{[4 \mathrm{c}]}$ it performed poorly in aqueous environment. Our initial trials using 1 at 40 bar of equimolar $\mathrm{H}_{2} / \mathrm{CO}_{2}$ in water in the presence of $\mathrm{KOH}$ base provided very low formate yields (Table 1, entries 3 and 6 ) with maximal TON of 1573 at $90{ }^{\circ} \mathrm{C}$. Assuming the low solubility of 1 to be the major factor limiting catalytic performance in the aqueous media, we 
performed $\mathrm{CO}_{2}$ hydrogenation tests in the presence of additional organic solvent. Both a water-miscible DMF and immiscible toluene addition could improve the formate yields (Table 1), however the $\mathrm{KOH}$ conversion remained limited to a maximum of $26 \%$. We envisioned that such limitation might stem from the poor transport of ionic species across the phase boundary and employed a phase-transfer catalyst (PTC, methyltrioctylammonium chloride) to enhance it. Addition of PTC resulted in a significant increase of formate yields in biphasic catalytic system (Table 2).

Table 1. Solvent effect on the hydrogenation of $\mathrm{CO}_{2}$ with $\mathrm{KOH}$ as the base. ${ }^{a}$

\begin{tabular}{l|l|l|l|l}
\hline Entry & Solvent & $\begin{array}{l}\mathrm{KOH} \\
(\mathrm{mmol})\end{array}$ & TON & Yield (\%) \\
\hline 1 & $\mathrm{H}_{2} \mathrm{O} / \mathrm{DMF}$ & 2.5 & 5627 & 26 \\
2 & $\mathrm{H}_{2} \mathrm{O} /$ toluene & 2.5 & 3125 & 13 \\
3 & $\mathrm{H}_{2} \mathrm{O}$ & 2.5 & 745 & 3.3 \\
4 & $\mathrm{H}_{2} \mathrm{O} / \mathrm{DMF}$ & 5.0 & 7610 & 18 \\
5 & $\mathrm{H}_{2} \mathrm{O} /$ toluene & 5.0 & 2992 & 7.5 \\
6 & $\mathrm{H}_{2} \mathrm{O}$ & 5.0 & 1573 & 3.7 \\
\hline
\end{tabular}

[a] Reaction conditions: $2 \mathrm{~mL}$ total solvent (1/1 in case of mixed solvent systems), $0.107 \mu \mathrm{mol}$ of catalyst $1, \mathrm{~T}=90^{\circ} \mathrm{C}, \mathrm{p}=40$ bar $\left(\mathrm{p}_{\mathrm{H} 2}=\mathrm{p}_{\mathrm{CO} 2}=20 \mathrm{bar}\right), \mathrm{t}$ $=3 \mathrm{~h}$.

Table 2. Effect of using methyltrioctylammonium chloride as a phase-transfer catalyst in the hydrogenation of $\mathrm{CO}_{2}$ in biphasic system, using $\mathrm{KOH}$ as a base. ${ }^{2}$

\begin{tabular}{l|l|l|l|l}
\hline Entry & PTC $(\mathrm{mmol})$ & $\begin{array}{l}\mathrm{KOH} \\
(\mathrm{mmol})\end{array}$ & TON & Yield $(\%)$ \\
\hline 1 & no & 2.5 & 3125 & 13 \\
2 & no & 5.0 & 2992 & 7.5 \\
3 & 0.025 & 5.0 & 20060 & 52 \\
4 & 0.29 & 5.0 & 17732 & 46 \\
5 & 0.052 & 14 & 16509 & 14 \\
\hline
\end{tabular}

[a] Reaction conditions: $2 \mathrm{~mL}$ total solvent $\left(\mathrm{H}_{2} \mathrm{O} /\right.$ toluene $\left.=1 / 1\right), 0.107 \mu \mathrm{mol}$ of catalyst $1, \mathrm{~T}=90{ }^{\circ} \mathrm{C}, \quad \mathrm{p}=40$ bar $\left(\mathrm{p}_{\mathrm{H} 2}=\mathrm{p}_{\mathrm{CO} 2}=20\right.$ bar $), \mathrm{PTC}$ is methyltrioctylammonium chloride, $\mathrm{t}=3 \mathrm{~h}$.

In the same screening experiment we noted that elevated base concentrations consistently resulted in lower formate yields, implying the limited stability of $\mathbf{1}$ in a highly alkaline medium (Table 2). Circumventing this, we opted to perform direct hydrogenation of bicarbonates - a process with additional advantage of using hydrogen as the only gaseous feedstock. Similar to $\mathrm{H}_{2} \mathrm{O} / \mathrm{KOH}$ system, the hydrogenation of $\mathrm{H}_{2} \mathrm{O} / \mathrm{KHCO}_{3}$ shows a limited efficiency in pure water or in the absence of a PTC (Table 3). However, a biphasic medium with PTC provides excellent TON values > 100000 with yields significantly higher than those found with potassium hydroxide base (Table 3 ).
Table 3. Hydrogenation of potassium bicarbonate, with and without using a phase-transfer catalyst. $^{\text {a }}$

\begin{tabular}{l|l|l|l|l|l}
\hline Entry & Solvent & $\begin{array}{l}\text { PTC } \\
(\mathrm{mmol})\end{array}$ & $\mathrm{KHCO}_{3}(\mathrm{mmol})$ & TON & Yield (\%) \\
\hline 1 & $\mathrm{H}_{2} \mathrm{O}$ & $\mathrm{No}$ & 10 & 3963 & 4.5 \\
2 & $\mathrm{H}_{2} \mathrm{O} /$ toluene & $\mathrm{No}$ & 10 & 4263 & 4.8 \\
3 & $\mathrm{H}_{2} \mathrm{O} /$ toluene & 0.055 & 10 & 66495 & 78 \\
4 & toluene & 0.055 & 5.0 & 289 & 0.6 \\
5 & $\mathrm{H}_{2} \mathrm{O} /$ toluene & 0.055 & 5.0 & 26897 & 65 \\
6 & $\mathrm{H}_{2}$ O/toluene & 0.055 & 14 & 102593 & 86 \\
\hline
\end{tabular}

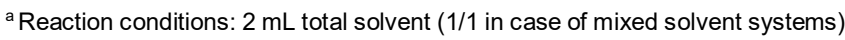
$0.107 \mu \mathrm{mol}$ of catalyst $1, \mathrm{~T}=90^{\circ} \mathrm{C}, \mathrm{p}=40$ bar $\left(\mathrm{p}_{\mathrm{H} 2}=40 \mathrm{bar}\right), \mathrm{t}=3 \mathrm{~h}, \mathrm{PTC}=$ methyltrioctylammonium chloride.

In sharp contrast with hydroxide bases, a gradual increase of $\mathrm{KHCO}_{3}$ loading leads to the marked increase in both observed TON and final formate yield. For example, we could utilize $\mathrm{KHCO}_{3}$ loadings of up to $14 \mathrm{mmol} / \mathrm{mL}_{\mathrm{H} 2 \mathrm{O}}$ and obtain final yields in extent of $86 \%$ corresponding to TON of 102593 (Table 3, entry 6). Remarkably, even at the temperature of $90^{\circ} \mathrm{C}$, reaction mixture is saturated in potassium bicarbonate with the main fraction of $\mathrm{KHCO}_{3}$ remaining solid. In line with solubility of potassium formate being significantly higher than that of bicarbonate our approach effectively transforms bicarbonate slurries to $>50 \%$ wt formate solutions with no need for product concentration.

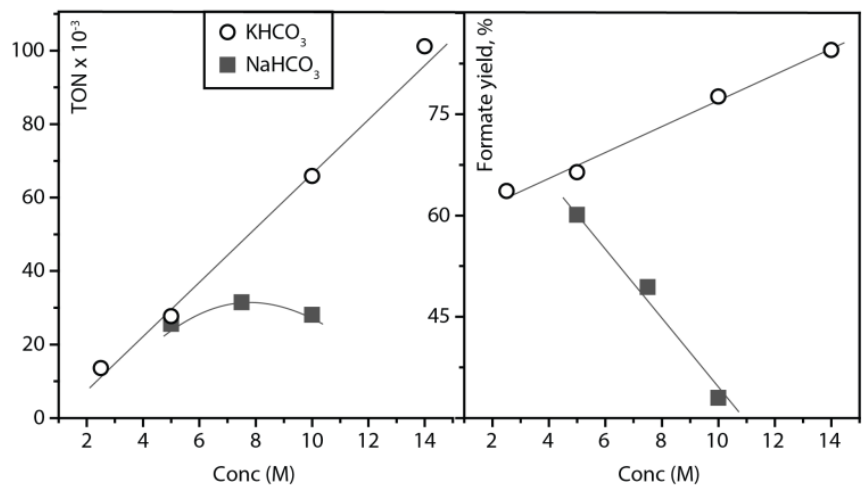

Figure 2. Concentration effects of $\mathrm{KHCO}_{3}$ and $\mathrm{NaHCO}_{3}$ on the formate yield and TONs. Reaction conditions: $2 \mathrm{~mL}$ solvent total $\left(\mathrm{H}_{2} \mathrm{O} /\right.$ toluene $\left.=1 / 1\right), 0.107$ $\mu \mathrm{mol}$ of catalyst $1,0.055 \mathrm{mmol}$ methyltrioctylammonium chloride, $\mathrm{T}=90^{\circ} \mathrm{C}, \mathrm{p}$ $=40 \mathrm{bar}\left(\mathrm{p}_{\mathrm{H} 2}=40 \mathrm{bar}\right), \mathrm{t}=4.5 \mathrm{~h}$, concentrations are considered as $\mathrm{M} / \mathrm{H}_{2} \mathrm{O}$.

The trend of increasing formate yield as a function of initial bicarbonate loading was confirmed in a separate study depicted in Figure 2. Upon increase of $\mathrm{KHCO}_{3}$ loading from 2 to $14 \mathrm{~mol}$ per litre water, the formate yield increased from 63 to $86 \%$. Interestingly, catalytic reaction apparently accelerated upon addition of bicarbonate as the registered TON values for hydrogenation also increased from 13373 to 102593 . Contrary to the case of potassium bicarbonate, $\mathrm{NaHCO}_{3}$ does not impact catalysis favourably upon the loading increase. Sodium formate yields decrease and TON values calculated as the number of turnovers per that of catalyst molecules remains similar. Considering that identical catalyst loadings were used for all 
experiments depicted in Figure 2, our data implies that selection of alkali cation is crucial for producing efficient catalytic system.

Finally, we examined the impact of reaction conditions on the performance of biphasic catalytic system. Varying the reaction temperature and pressure over in series of experiments (Figure 3 and ESI) we confirmed that biphasic hydrogenation exhibits behavior similar to that of single solvent systems. Namely, the increase of reaction temperature from 65 to $120{ }^{\circ} \mathrm{C}$ leads to the marked drop in formate yield, while the increase of $\mathrm{H}_{2}$ pressure from 5 to 60 bar $\mathrm{H}_{2}$ results in the increased formate yield. This is consistent with expected thermodynamic behavior of the system as well as recent reports detailing this reaction. ${ }^{[12]}$

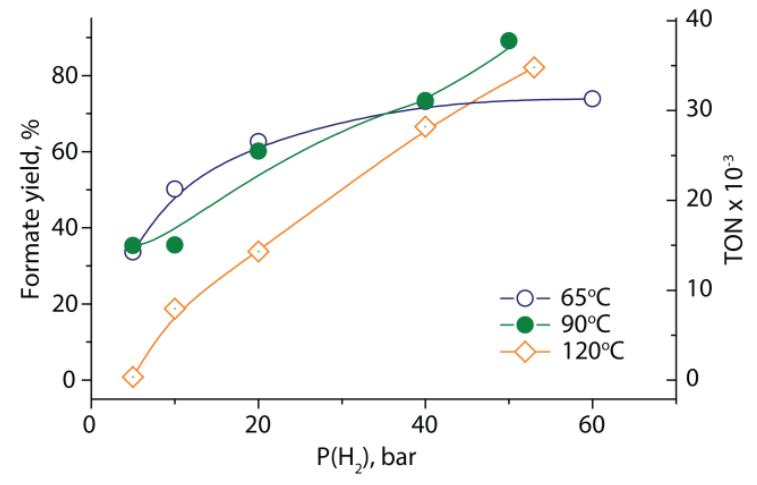

Figure 3. Effect of temperature and pressure on the formate yield and TONs in the hydrogenation of $\mathrm{KHCO}_{3}$. Reaction conditions: $2 \mathrm{~mL}$ solvent, $0.107 \mu \mathrm{mol}$ of catalyst $1,55 \mathrm{mM}$ methyltrioctylammonium chloride, $\mathrm{t}=16 \mathrm{~h}, \mathrm{KHCO}_{3}=5 \mathrm{mmol}$, $T$ and $p$ are varied.

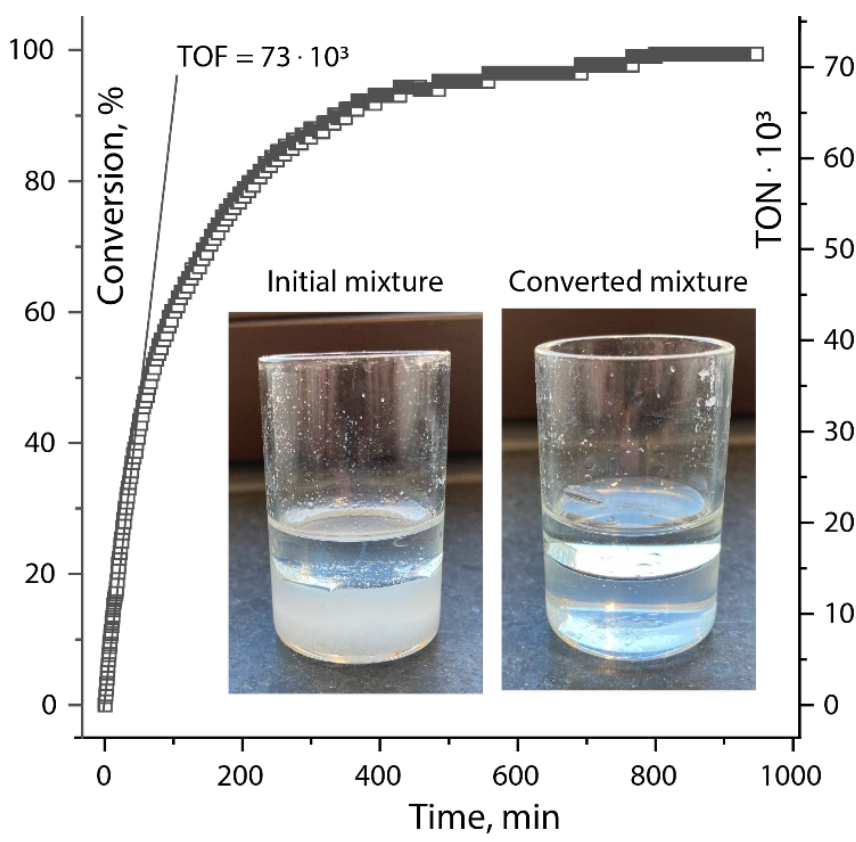

Figure 4. Conversion versus time in the hydrogenation of $\mathrm{KHCO}_{3}$. Reaction conditions: $10 \mathrm{~mL}$ solvent $\left(\mathrm{H}_{2} \mathrm{O} /\right.$ toluene $\left.=1 / 1\right), 0.535 \mu \mathrm{mol}$ of catalyst 1 , methyltrioctylammonium chloride, $\mathrm{T}=90^{\circ} \mathrm{C}, \mathrm{p}=50$ bar $\left(\mathrm{p}_{2}=50\right.$ bar), $\mathrm{KHCO}_{3}$ $=50 \mathrm{mmol}\left(10 \mathrm{M}\right.$ in $\left.\mathrm{H}_{2} \mathrm{O}\right)$. Inside the graph are the stirred reaction mixtures before (left) and after (right) reaction, showing the net conversion of the bicarbonate suspension into a clear solution at RT.
Examining kinetics of bicarbonate hydrogenation we found that $\mathbf{1}$ is capable of developing high turnover frequencies (TOF) with no apparent inhibition often observed in organic media. ${ }^{[13]}$ Tracking hydrogen consumption in the course of hydrogenation we estimated initial TOF in extent of $73000 \mathrm{~h}^{-1}$ (Figure 4) with kinetic traces following a regular monoexponential trendline. These experiments could also confirm the ease of scaling this reaction that was performed on $50 \mathrm{mmol}$ scale as compared to a five-fold lower scale used in screening.

Finally, we probed the performance limits of biphasic catalytic system based on 1. In order to maximize TON per batch we varied the catalyst concentration and found no significant productivity drop with catalyst concentration as low as $2 \mathrm{ppm}$ with respect to bicarbonate substrate (Table 4). Under these conditions, TON values up to 300000 can be achieved with similar formate yields in extent of $70 \%$, producing clear formate solutions at the end of the catalytic reaction.

Table 4. Effect of the concentration of catalyst on the final yield and TON.

\begin{tabular}{l|l|l|l}
\hline Entry & Cat $(\mu \mathrm{mol})$ & TON & Yield $(\%)$ \\
\hline 1 & 0.006 & 474026 & 55 \\
2 & 0.012 & 296756 & 70 \\
3 & 0.029 & 109224 & 64 \\
4 & 0.059 & 56996 & 67 \\
5 & 0.12 & 27728 & 67 \\
\hline
\end{tabular}

[a] Reaction conditions: $2 \mathrm{~mL}$ solvent, $5 \mathrm{mmol} \mathrm{KHCO}_{3}, \mathrm{~T}=90^{\circ} \mathrm{C}, \mathrm{p}_{2}=40 \mathrm{bar}$, $0.055 \mathrm{mmol}$ methyltrioctylammonium chloride, $\mathrm{t}=18 \mathrm{~h}$.

To conclude, in this work we developed a potent catalytic system based on ruthenium complex 1. Making use of its biphasic composition, our system is capable of converting supersaturated bicarbonate slurries to formate solutions close to their saturation point. We demonstrate generation of ca. $50 \%_{\text {wt }}$ aqueous formate solutions from commercial potassium bicarbonate with no substrate purification required prior to catalysis. With a maximum TON of $474 \times 10^{3}$ catalyst 1 appears very promising for developing intense hydrogenation processes in aqueous medium free of the limitations associated with conventional organic solvents.

\section{Acknowledgements}

This research was carried out with the financial support from Nitto Denko Corporation.

Keywords: bicarbonate $\cdot$ catalysis $\bullet$ hydrogenation $\cdot$ formate $\cdot$ multiphasic

[1] a) P. G. Jessop, T. Ikariya, R. Noyori, Nature 1994, 368, 231-233; b) Q. Liu, L. Wu, R. Jackstell, M. Beller, Nat. Commun. 2015, 6, 5933.

[2] aM.-Y. Lee, K. T. Park, W. Lee, H. Lim, Y. Kwon, S. Kang, Critical Rev.Environ. Sci. Tech. 2020, 50, 769-815; b) D. H. Apaydin, S Schlager, E. Portenkirchner, N. S. Sariciftci, ChemPhysChem 2017 , 18, 3094-3116

[3] Y. Himeda, CO2 Hydrogenation Catalysis, V. C. H. Wiley, 2021. 

Muckerman, E. Fujita, Y. Himeda, Inorg. Chem. 2015, 54, 5114 5123; c) G. A. Filonenko, R. van Putten, E. N. Schulpen, E. J. M. Hensen, E. A. Pidko, Chem CatChem 2014, 6, 1526-1530; d) E. Graf, W. Leitner, J. Chem. Soc. Chem. Comm. 1992, 623-624; e) H. Hayashi, S. Ogo, S. Fukuzumi, Chem. Commun. 2004, 2714-2715 f) Y. Himeda, N. Onozawa-Komatsuzaki, H. Sugihara, H. Arakawa, K. Kasuga, Organometallics 2004, 23, 1480-1483; g) Y. Himeda, N. Onozawa-Komatsuzaki, H. Sugihara, K. Kasuga, Organometallics 2007, 26, 702-712; h) C. A. Huff, M. S. Sanford, ACS Catal. 2013 3, 2412-2416; i) D. Jantke, L. Pardatscher, M. Drees, M. Cokoja, W. A. Herrmann, F. E. Kühn, ChemSusChem 2016, 9, 2849-2854; j) P G. Jessop, Y. Hsiao, T. Ikariya, R. Noyori, J. Am. Chem. Soc. 1996 118, 344-355; k) F. Joó, F. Joó, L. Nádasdi, J. Elek, G. Laurenczy, L. Nádasdi, Chem. Commun. 1999, 971-972; I) J. Kothandaraman M. Czaun, A. Goeppert, R. Haiges, J.-P. Jones, R. B. May, G. K. S Prakash, G. A. Olah, ChemSusChem 2015, 8, 1442-1451; m) S.-M. Lu, Z. Wang, J. Li, J. Xiao, C.Li, Green Chem. 2016, 18, 4553-4558; n) Y. Maenaka, T. Suenobu, S. Fukuzumi, Energy Environ. Sc. 2012 5, 7360-7367; o) S. Oldenhof, J. I. van der Vlugt, J. N. H. Reek, Catal. Sci. Technol. 2016, 6, 404-408; p) T. J. Schmeier, G. E Dobereiner, R. H. Crabtree, N. Hazari, J. Am. Chem. Soc. 2011, 133, 9274-9277; q) R. Tanaka, M. Yamashita, K. Nozaki, J. Am. Chem. Soc. 2009, 131, 14168-14169.

[5] a) C. Federsel, A. Boddien, R. Jackstell, R. Jennerjahn, P. J. Dyson R. Scopelliti, G. Laurenczy, M. Beller, Angew. Chem. Int. Ed. 2010 49, 9777-9780; b) R. Langer, Y. Diskin-Posner, G. Leitus, L. J. W Shimon, Y. Ben-David, D. Milstein, Angew. Chem. Int. Ed. 2011, 50 9948-9952; c) Y. Zhang, A. D. Maclntosh, J. L. Wong, E. A. Bielinski, P. G. Williard, B. Q. Mercado, N. Hazari, W. H. Bernskoetter, Chem. Sci. 2015, 6, 4291-4299; d) C. Ziebart, C. Federsel, P. Anbarasan, R. Jackstell, W. Baumann, A. Spannenberg, M. Beller, J. Am. Chem. Soc. 2012, 134, 20701-20704; e) F. Bertini, N. Gorgas, B. Stöger M. Peruzzini, L. F. Veiros, K. Kirchner, L. Gonsalvi, ACS Catal. 2016 6, 2889-2893

[6] a) J. Choi, Y. Lee, Inorg. Chem. Frontiers 2020, 7, 1845-1850; b) C. Federsel, C. Ziebart, R. Jackstell, W. Baumann, M. Beller, Chem. Eur. J. 2012, 18, 72-75; c) M. S. Jeletic, M. T. Mock, A. M. Appel, J. C. Linehan, J. Am. Chem. Soc. 2013, 135, 11533-11536; d) J. Schneidewind, R. Adam, W. Baumann, R. Jackstell, M. Beller, Angew. Chem. Int. Ed. 2017, 56, 1890-1893.

[7] a) F. Bertini, M. Glatz, N. Gorgas, B. Stöger, M. Peruzzini, L. F. Veiros, K. Kirchner, L. Gonsalvi, Chem. Sci. 2017, 8, 5024-5029; b) A. Dubey, L. Nencini, R. R. Fayzullin, C. Nervi, J. R. Khusnutdinova ACS Catal. 2017, 7, 3864-3868; c) S. Kar, A Goeppert, J. Kothandaraman, G. K. S. Prakash, ACS Catal. 2017, 7, 6347-6351. a) R. Kanega, M. Z. Ertem, N. Onishi, D. J. Szalda, E. Fujita, Y Himeda, Organometallics 2020, 39, 1519-1531; b) S. Xu, N. Onishi, A. Tsurusaki, Y. Manaka, W.-H. Wang, J. T. Muckerman, E. Fujita, Y. Himeda, Eur. J.Inorg. Chem. 2015, 5591-5594.

[9] B. Chen, M. Dong, S. Liu, Z. Xie, J. Yang, S. Li, Y. Wang, J. Du, H. Liu, B. Han, ACS Catal. 2020, 10, 8557-8566.

[10] a) Z. Li, T. M. Rayder, L. Luo, J. A. Byers, C.-K. Tsung, J. Am. Chem. Soc. 2018, 140, 8082-8085; b) C. Wu, F. Irshad, M. Luo, Y. Zhao, X. Ma, S. Wang, ChemCatChem 2019, 11, 1256-1263

[11] a) M. Scott, B. Blas Molinos, C. Westhues, G. Franciò, W. Leitner Chem SusChem 2017, 10, 1085-1093; b) C. Guan, Y. Pan, E. P. L. Ang, J. Hu, C. Yao, M.-H. Huang, H. Li, Z. Lai, K.-W. Huang, Green Chem. 2018, 20, 4201-4205.

[12] A. Weilhard, S. P. Argent, V. Sans, Nat. Commun. 2021, 12, 231

[13] G. A. Filonenko, M. P. Conley, C. Copéret, M. Lutz, E. J. M. Hensen, E. A. Pidko, ACS Catal. 2013, 3, 2522-2526. 


\section{SUPPORTING INFORMATION}

\section{Homogeneous hydrogenation of saturated bicarbonate slurry to formates using multiphase catalysis}

Christophe Rebreyend, ${ }^{a}$ Evgeny A. Pidko* and Georgy A. Filonenko ${ }^{a *}$

[a] Inorganic Systems Engineering group, Department of Chemical Engineering, Delft University of Technology, The Netherlands

Corresponding authors: $\quad$ Evgeny A. Pidko (e.a.pidko@tudelft.nl)

Georgy A. Filonenko (g.a.filonenko@tudelft.nl) 


\section{S1. General considerations}

All manipulations were, unless stated otherwise, performed under inert atmosphere in an argon filled glovebox (INERT) or using standard Schlenk techniques. Anhydrous solvents were either dispensed from an Inert PureSolv solvent purification system or dried using 3/4 A molecular sieves and were degassed before use. Chemicals were purchased from Sigma-Aldrich, Strem, abcr or TCI. Air and/or moisture sensitive materials were stored in the glovebox. Deuterated solvents were purchased from Eurisotop, dried using molecular sieves, degassed and stored in the glovebox.

NMR spectra were recorded on an Agilent 400-MR DD2 $400 \mathrm{MHz}$ spectrometer equipped with a $5 \mathrm{~mm}$ ONE NMR probe. All ${ }^{13} \mathrm{C}$ and ${ }^{31} \mathrm{P}$ NMR spectra were recorded with ${ }^{1} \mathrm{H}$ decoupling. All chemical shifts were referenced to residual solvent peaks $\left[\mathrm{D}_{2} \mathrm{O}: 4.79 \mathrm{ppm}\left({ }^{1} \mathrm{H}\right), \mathrm{CDCl}_{3}: 7.26 \mathrm{ppm}\left({ }^{1} \mathrm{H}\right)\right.$, $\left.77.2 \mathrm{ppm}\left({ }^{13} \mathrm{C}\right)\right]$.

Complex 1 was prepared according to a literature procedure. ${ }^{\mathrm{i}}$

\section{S2. General considerations}

\section{General procedure for catalytic hydrogenation of $\mathrm{CO}_{2}$ in presence of $\mathrm{KOH}$}

Stock solutions of $\mathbf{1}(0.012 \mathrm{M})$ were prepared in dimethylformamide solvent. In a typical run, potassium hydroxide, methyltrioctylammonium chloride $(24 \mathrm{mg}, 55 \mu \mathrm{mol})$, toluene $(1 \mathrm{~mL})$, water $(1$ $\mathrm{mL}$ ) and appropriate amount of the stock solution of complex 1 were combined in this order in a $4 \mathrm{~mL}$ glass vial equipped with a rare-earth stirring bar and transferred into a stainless steel autoclave in the glovebox. The system was purged with argon $(5 \times 8$ bar $)$ and $\mathrm{H}_{2}(7 \times 10$ bar $)$, pressurized with $\mathrm{H}_{2}$ to 3 bar, and heated to specified temperature, after which the $\mathrm{H}_{2}$ and $\mathrm{CO}_{2}$ were regulated to the desired pressure. After the desired reaction time, the autoclave was cooled and the pressure released, after which DMSO was added as an internal standard (100 $\mu \mathrm{L}, 1.408 \mathrm{mmol})$. A $100 \mu \mathrm{L}$ aliquot of the $\mathrm{H}_{2} \mathrm{O}$ layer was dissolved in $\mathrm{D}_{2} \mathrm{O}$ and the yield determined by ${ }^{1} \mathrm{H}$ NMR analysis.

\section{General procedure for catalytic hydrogenation of potassium bicarbonate}

Stock solutions of $\mathbf{1}(0.012 \mathrm{M})$ were prepared in dimethylformamide solvent. In a typical run, potassium bicarbonate, methyltrioctylammonium chloride $(24 \mathrm{mg}, 55 \mu \mathrm{mol})$, toluene $(1 \mathrm{~mL})$, water $(1$ $\mathrm{mL}$ ) and appropriate amount of the stock solution of complex 1 were combined in this order in a $4 \mathrm{~mL}$ glass vial equipped with a rare-earth stirring bar and transferred into a stainless steel autoclave in the glovebox. The system was purged with argon $(5 \times 8$ bar $)$ and $\mathrm{H}_{2}(7 \times 10$ bar $)$, pressurized with $\mathrm{H}_{2}$ to 5 bar, and heated to specified temperature, after which the $\mathrm{H}_{2}$ was regulated to the desired pressure. After the desired reaction time, the autoclave was cooled and the pressure released, after which DMSO 
was added as an internal standard $(100 \mu \mathrm{L}, 1.408 \mathrm{mmol})$. A $100 \mu \mathrm{L}$ aliquot of the $\mathrm{H}_{2} \mathrm{O}$ layer was dissolved in $\mathrm{D}_{2} \mathrm{O}$ and the yield determined by ${ }^{1} \mathrm{H}$ NMR analysis. 
${ }^{1} \mathrm{H}$ NMR data accompanying data provided in Table 1

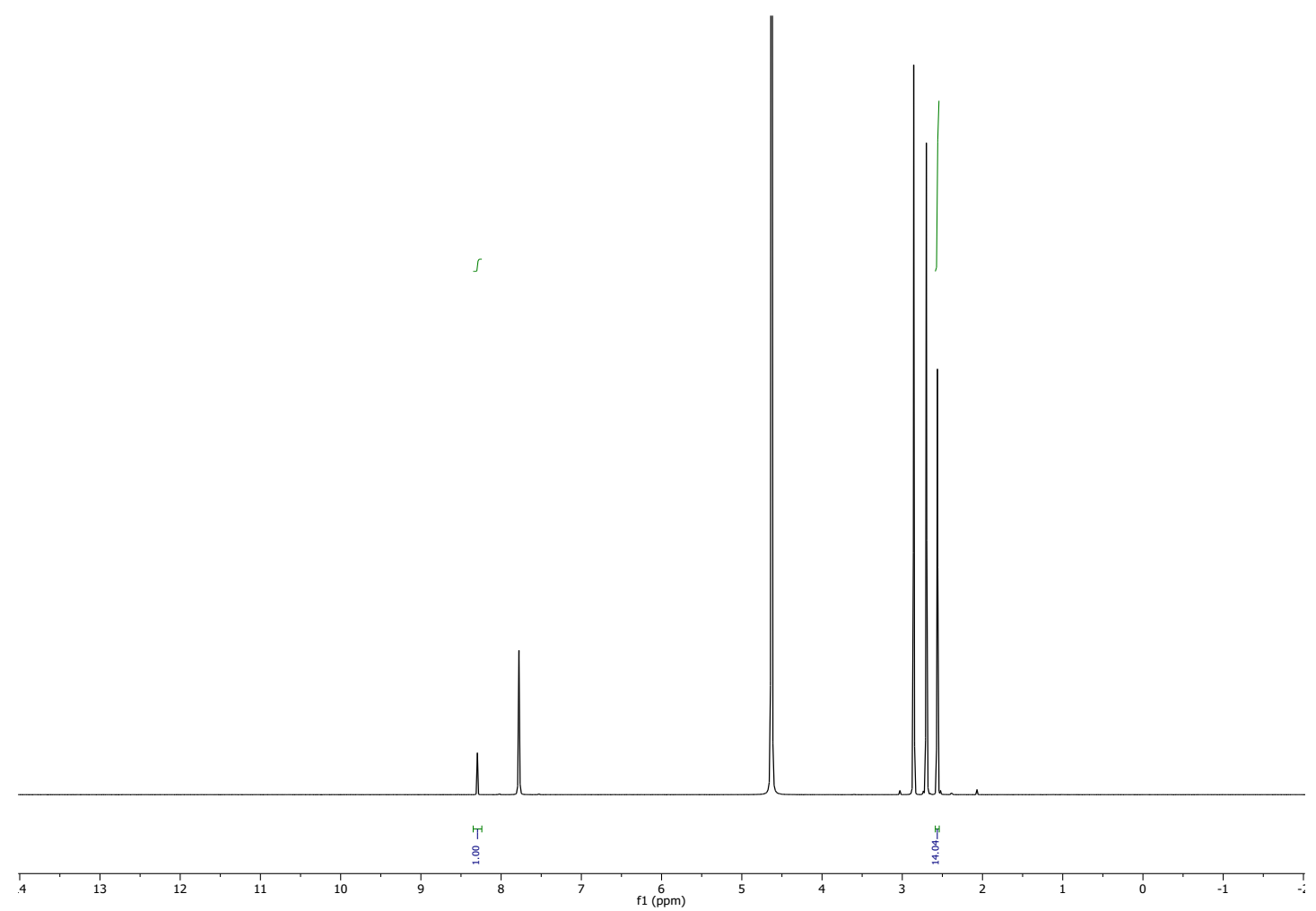

Figure S1. NMR data belonging to table 1, entry 1 .

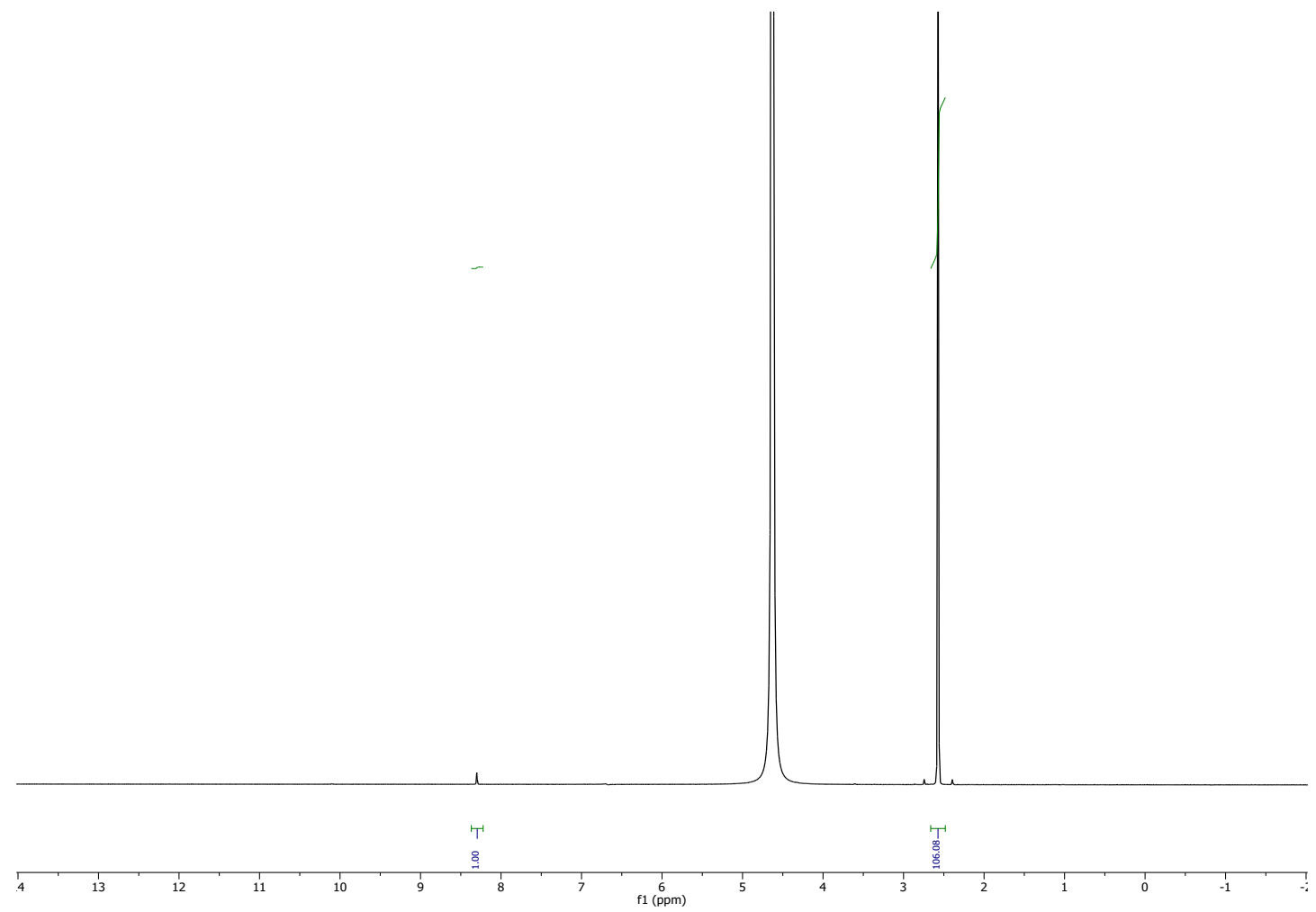

Figure S2. NMR data belonging to table 1, entry 2 . 


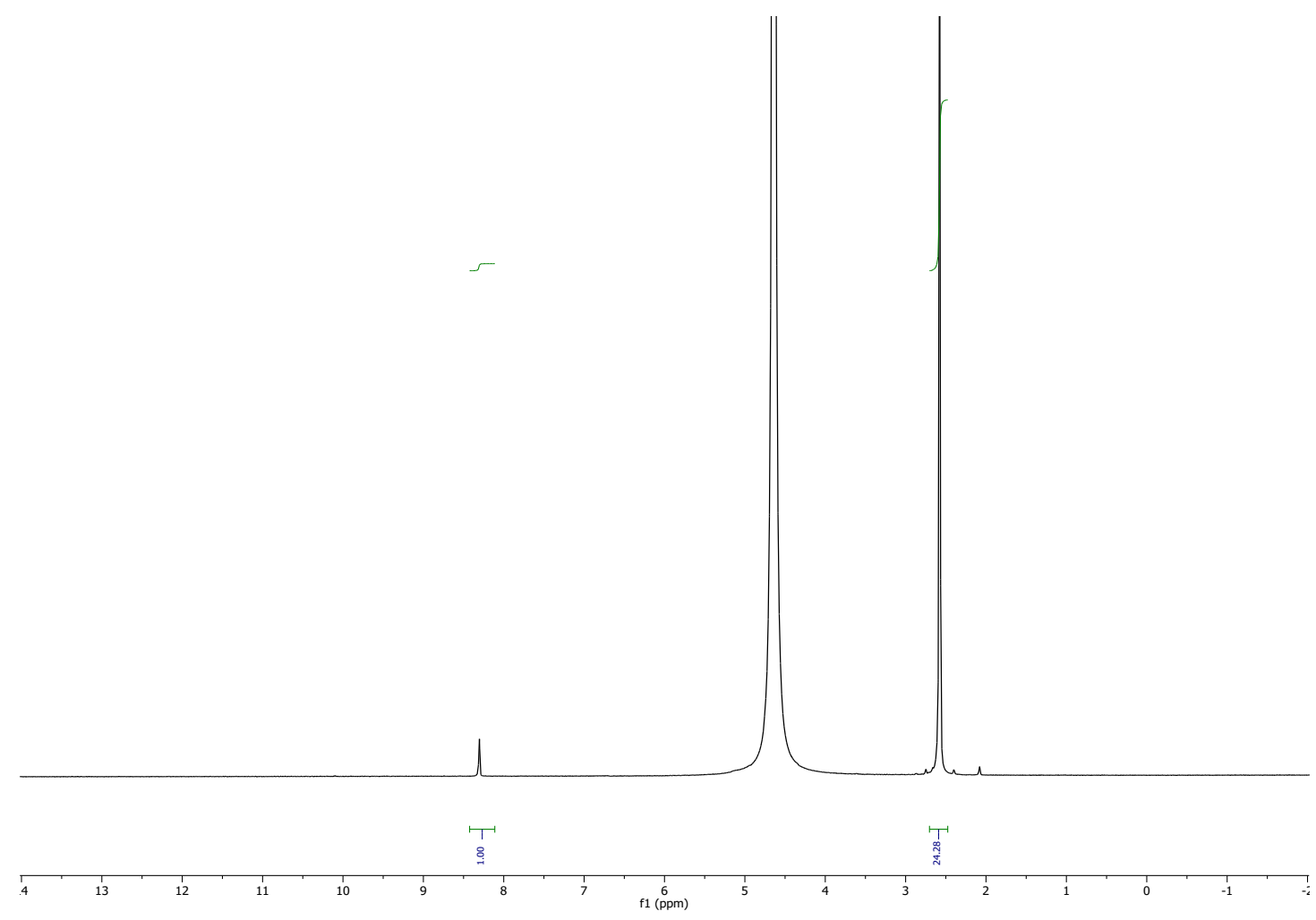

Figure S3. NMR data belonging to table 1, entry 3 .

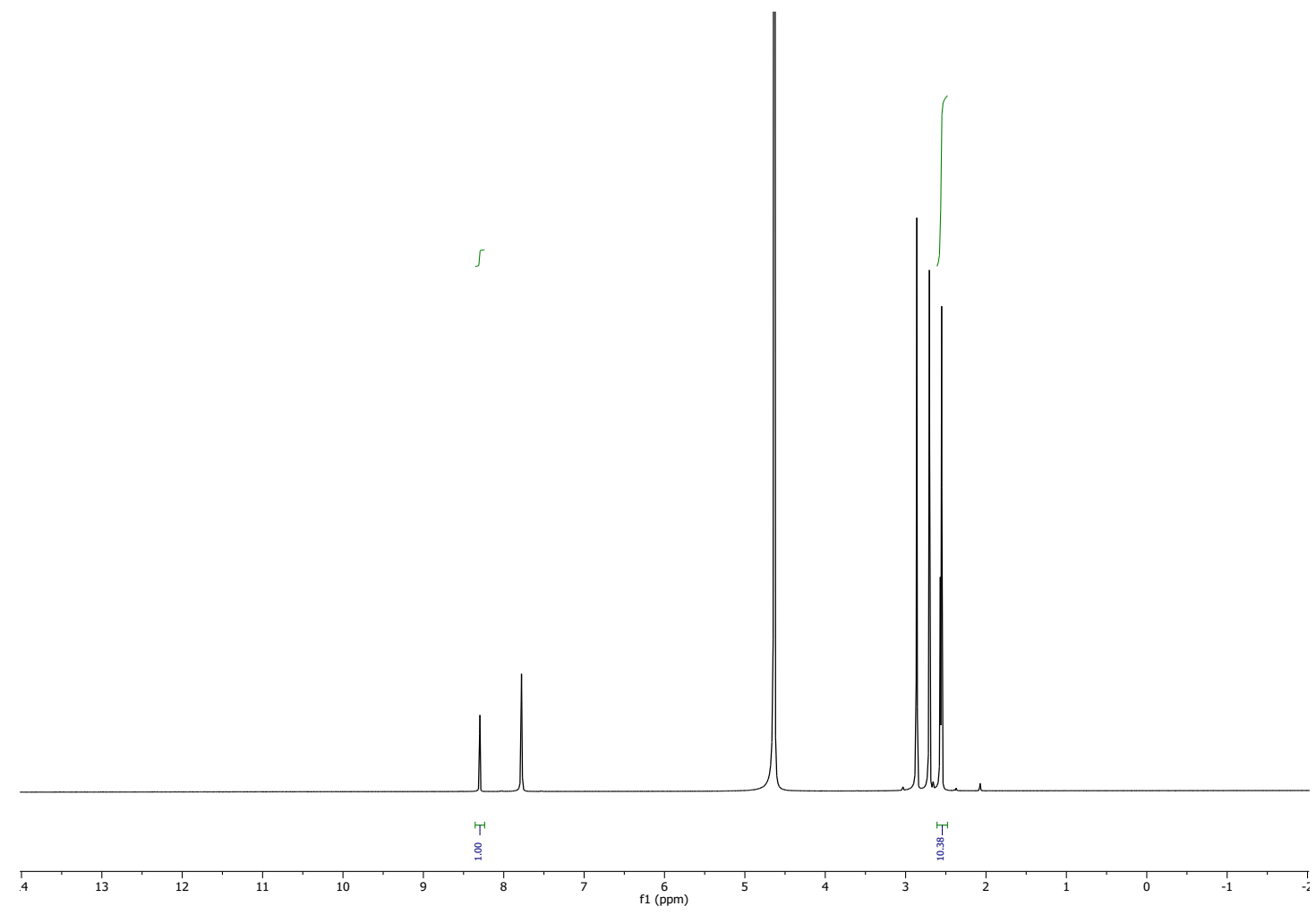

Figure S4. NMR data belonging to table 1, entry 4 . 


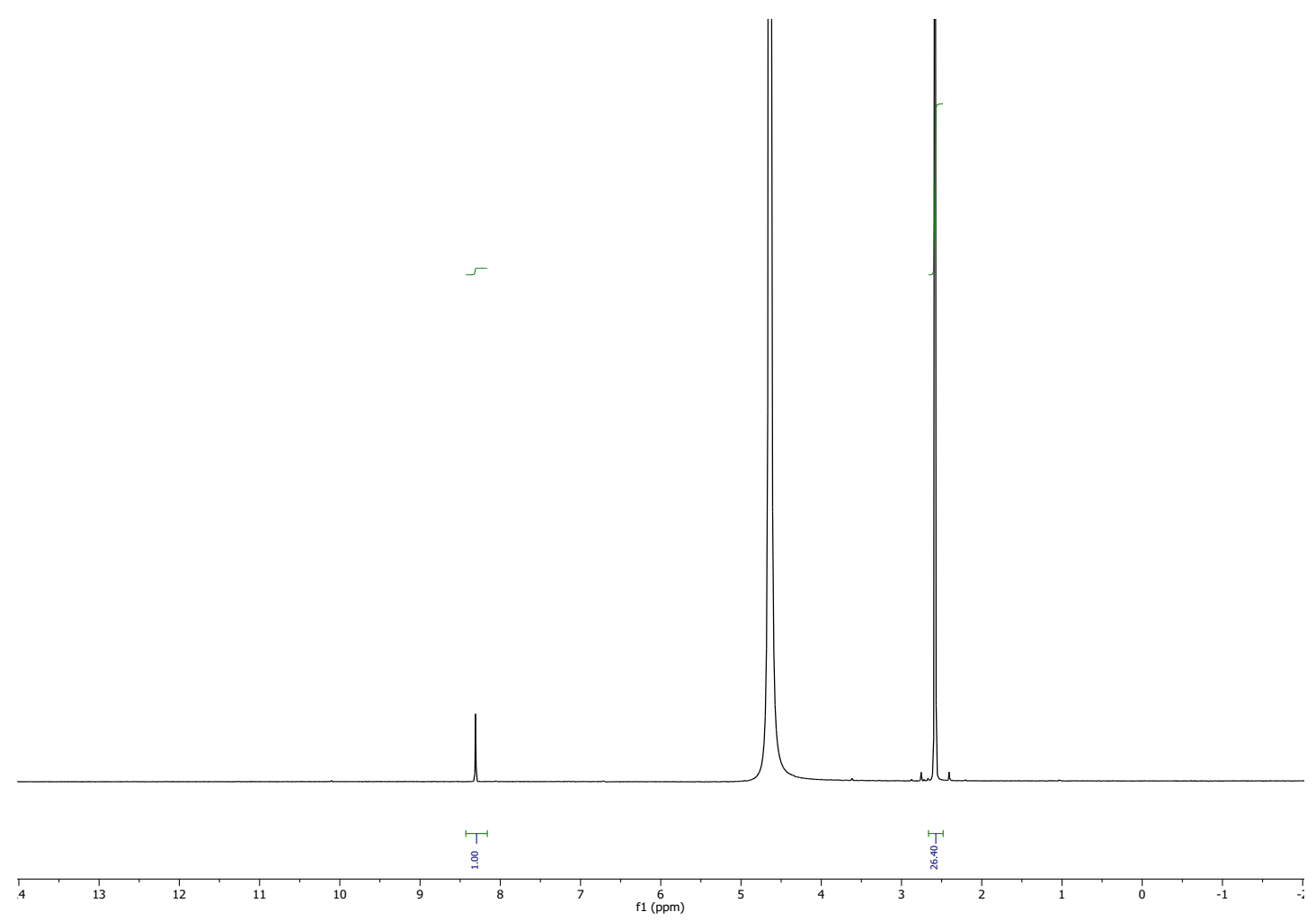

Figure S5. NMR data belonging to table 1, entry 5.

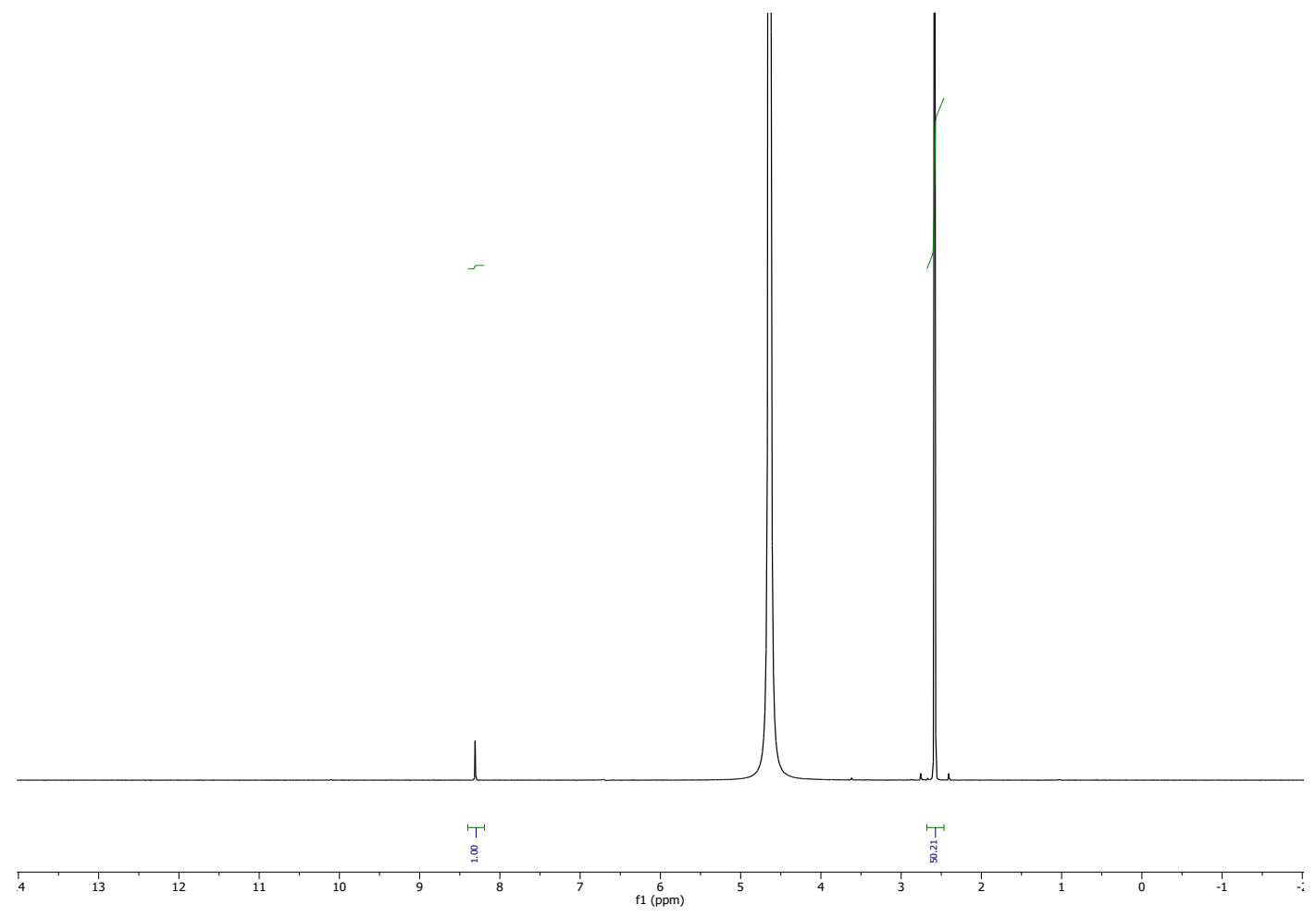

Figure S6. NMR data belonging to table 1, entry 6 . 
Table S1. ${ }^{1} \mathrm{H}$ NMR peak data belonging to table 1 (main text) and Figures S1-S6.

\begin{tabular}{|l|l|}
\hline Entry & Ratio of DMSO to formate integral \\
\hline 1 & 14.04 \\
\hline 2 & 24.28 \\
\hline 3 & 106.08 \\
\hline 4 & 10.38 \\
\hline 5 & 26.40 \\
\hline 6 & 50.21 \\
\hline
\end{tabular}


${ }^{1}$ H NMR data accompanying data provided in Table 2

NB: Entries 1-2 are covered in Figure S3 and Figure S6.

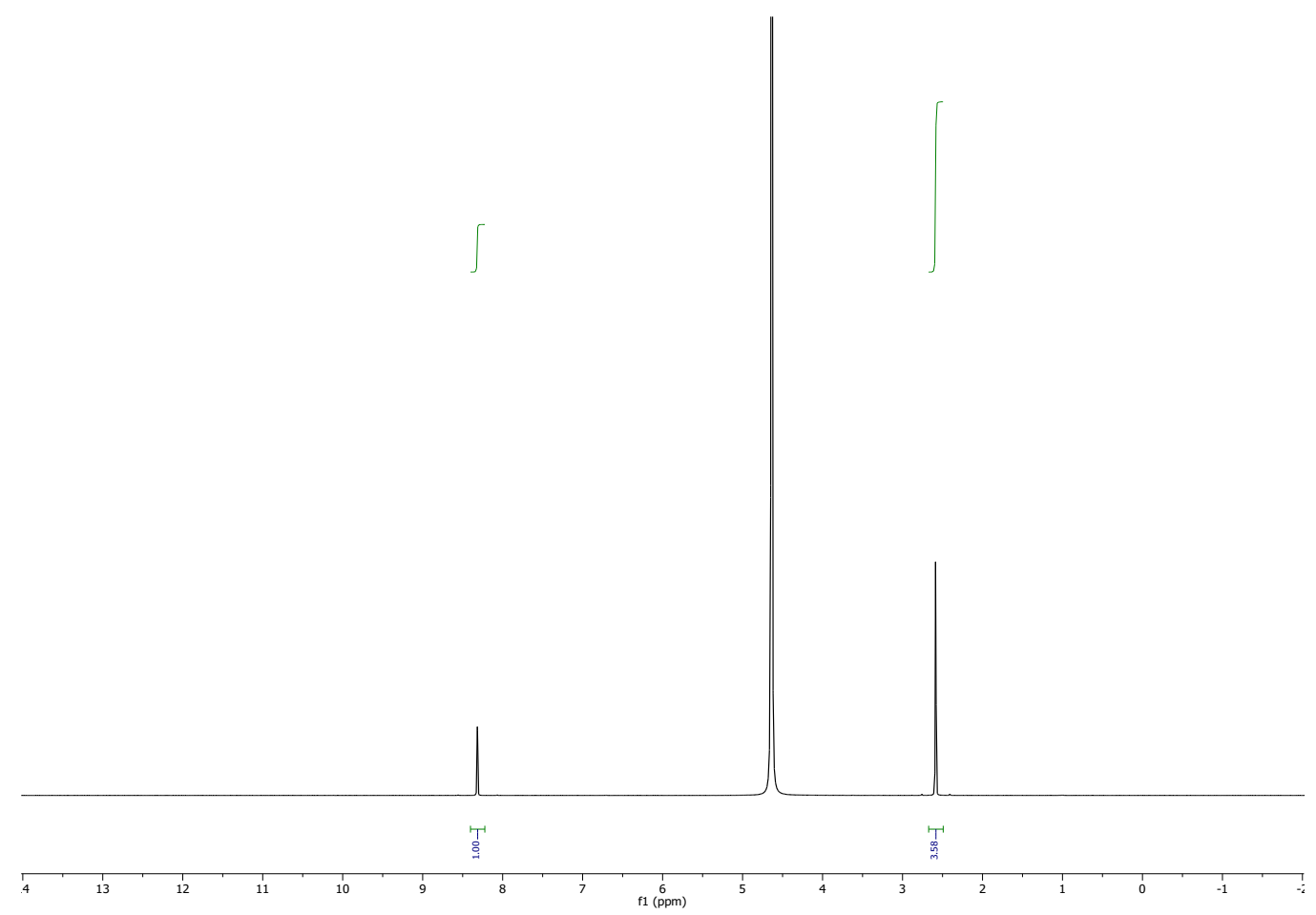

Figure S7. NMR data belonging to table 2, entry 3.

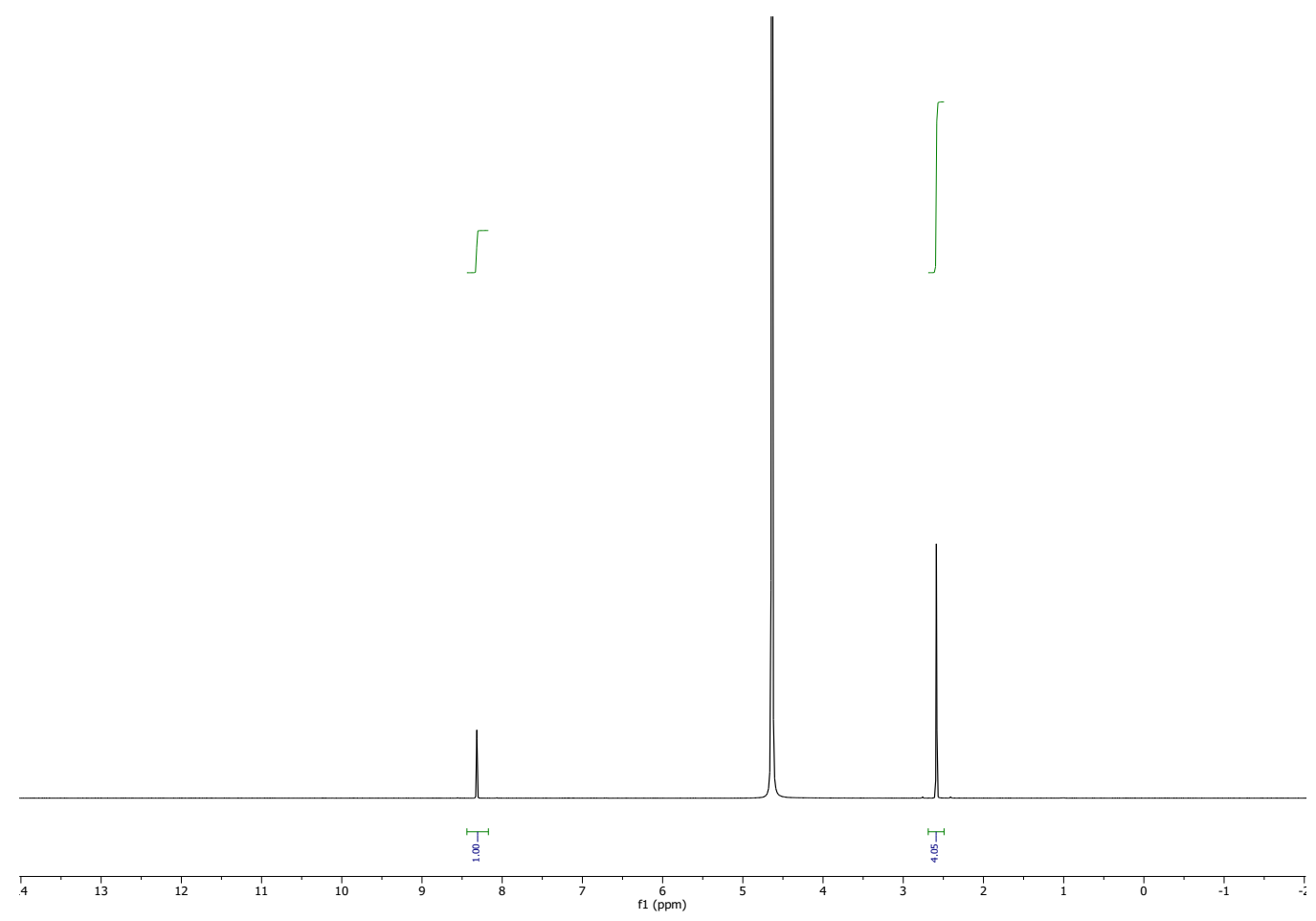

Figure S8. NMR data belonging to table 2, entry 4 . 


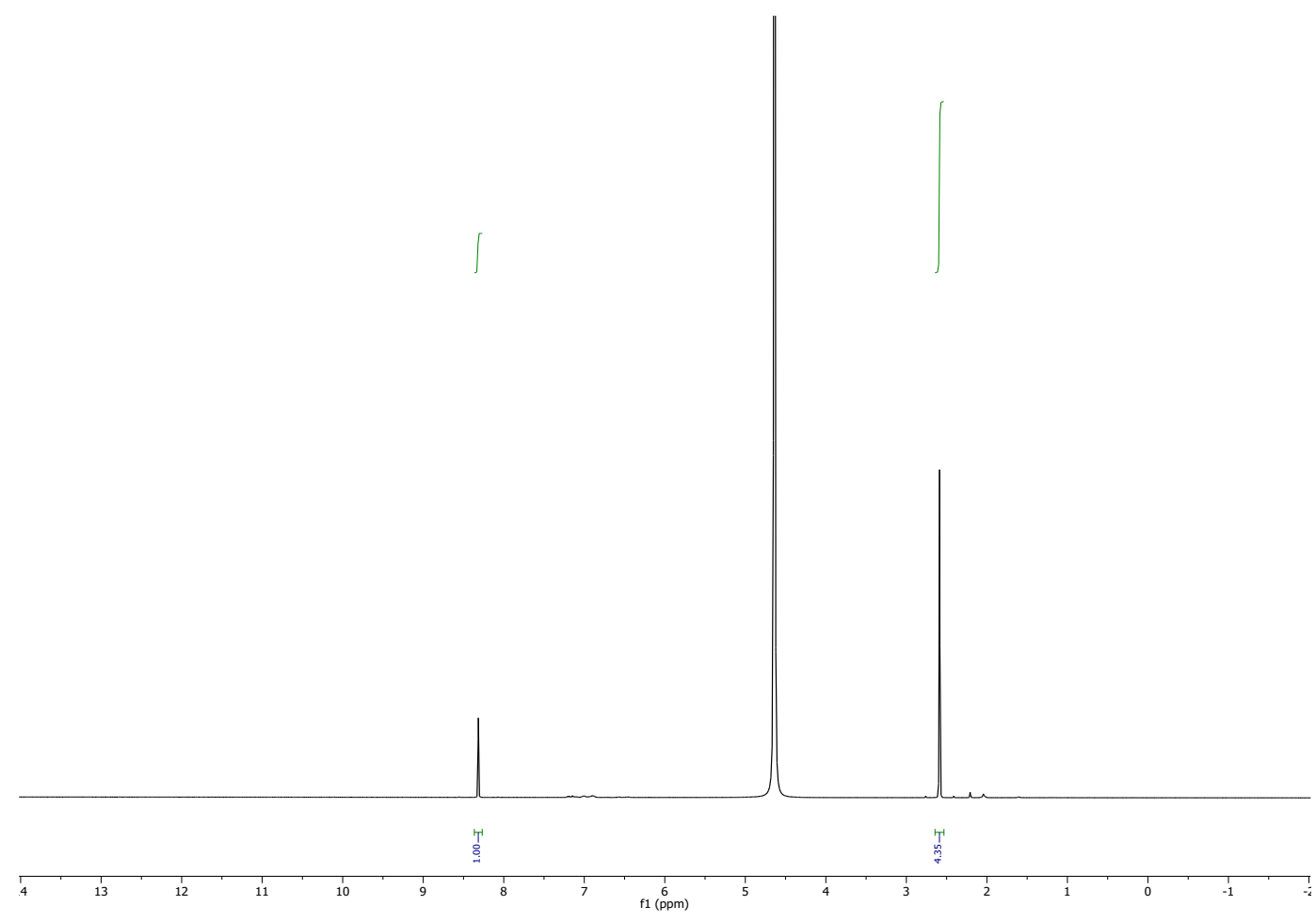

Figure S8. NMR data belonging to table 2, entry 5.

Table S2. ${ }^{1} \mathrm{H}$ NMR peak data belonging to table 2 (main text) and Figures S3 and S6-8.

\begin{tabular}{|l|l|}
\hline Entry & Ratio of DMSO to formate integral \\
\hline 1 & 106.08 \\
\hline 2 & 50.21 \\
\hline 3 & 3.58 \\
\hline 4 & 4.35 \\
\hline 5 & 4.05 \\
\hline
\end{tabular}


${ }^{1}$ H NMR Data belonging to table 3 (main text)

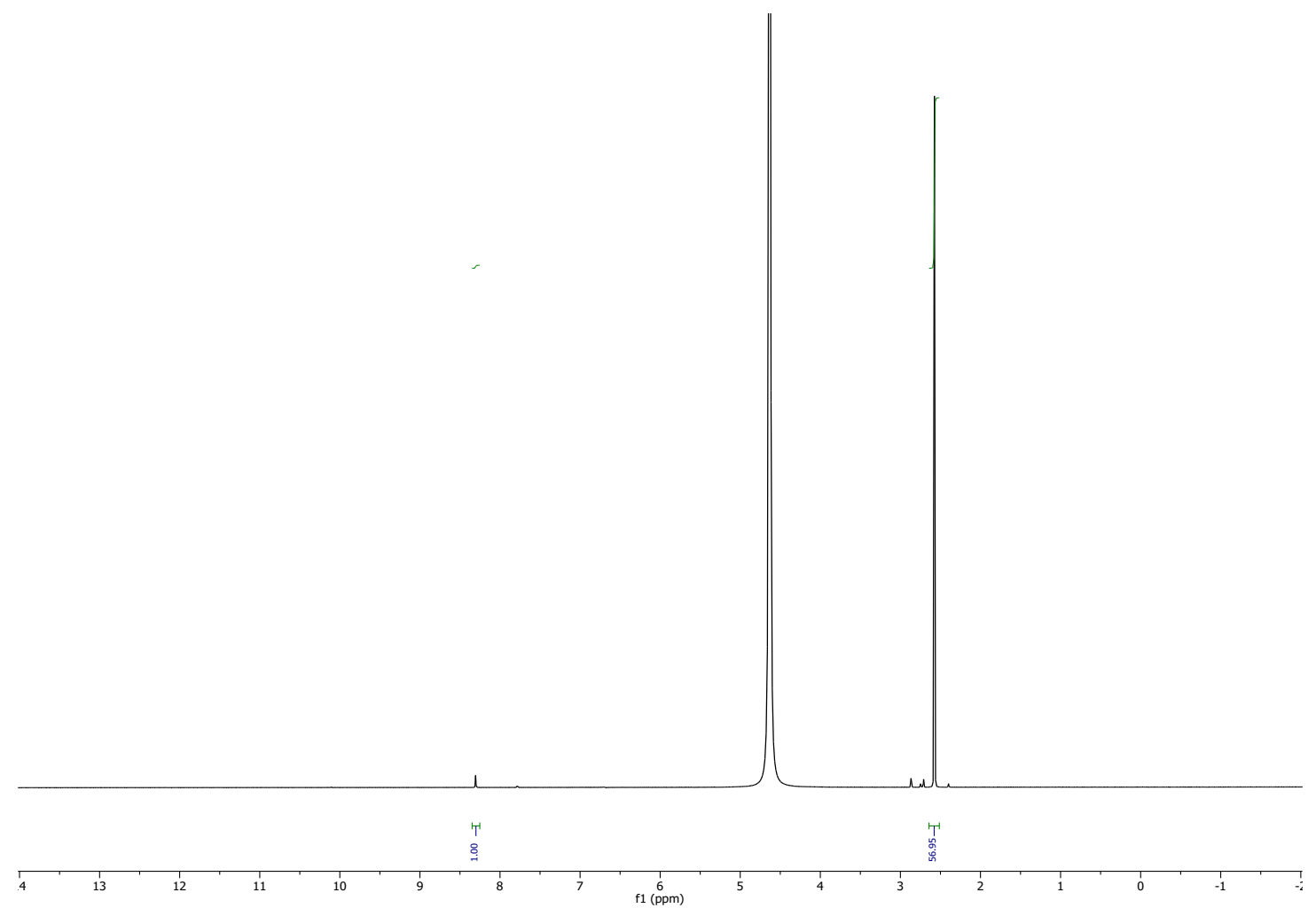

Figure S9. NMR data belonging to table 3, entry 1 .

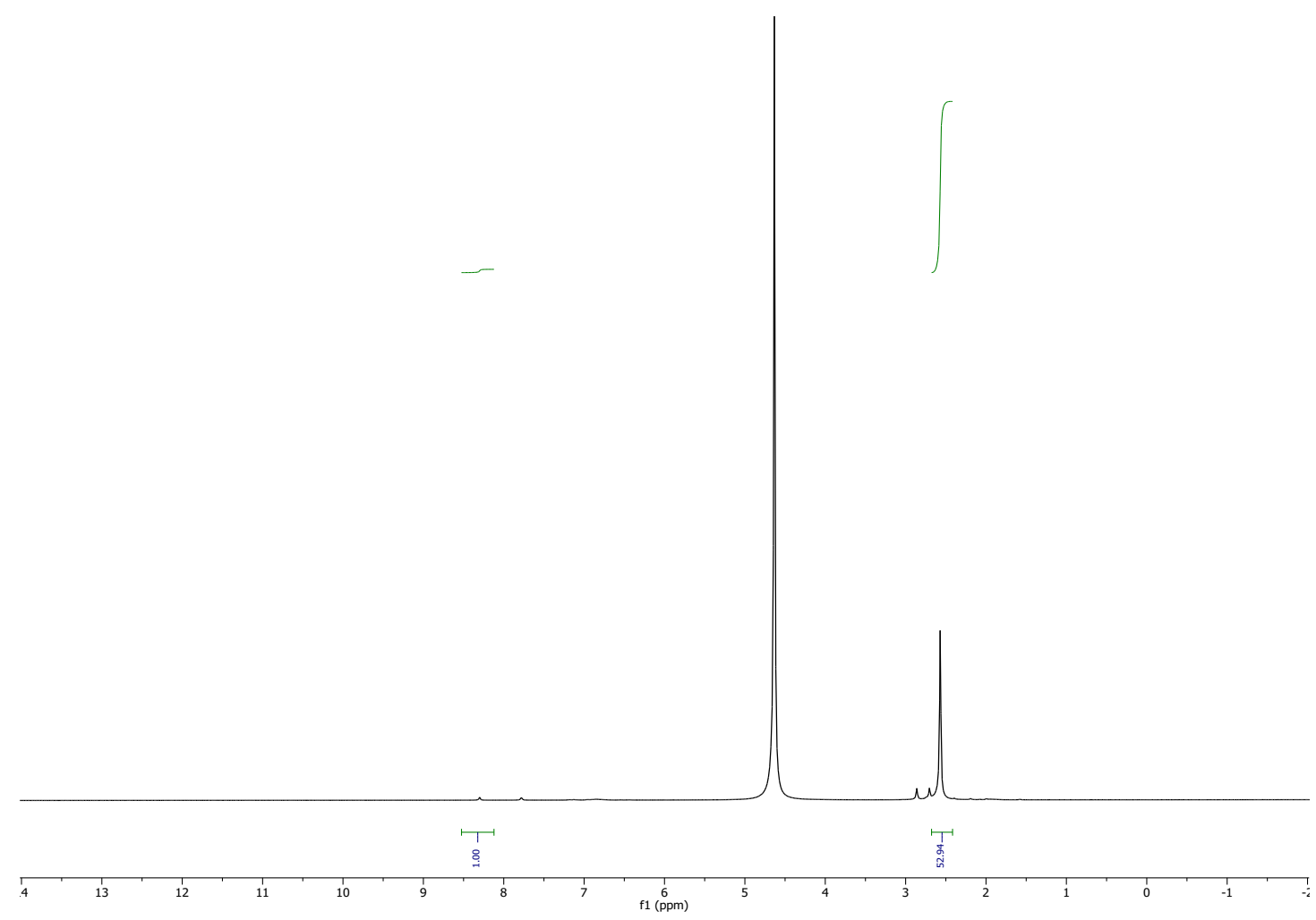

Figure S10. NMR data belonging to table 3, entry 2. 


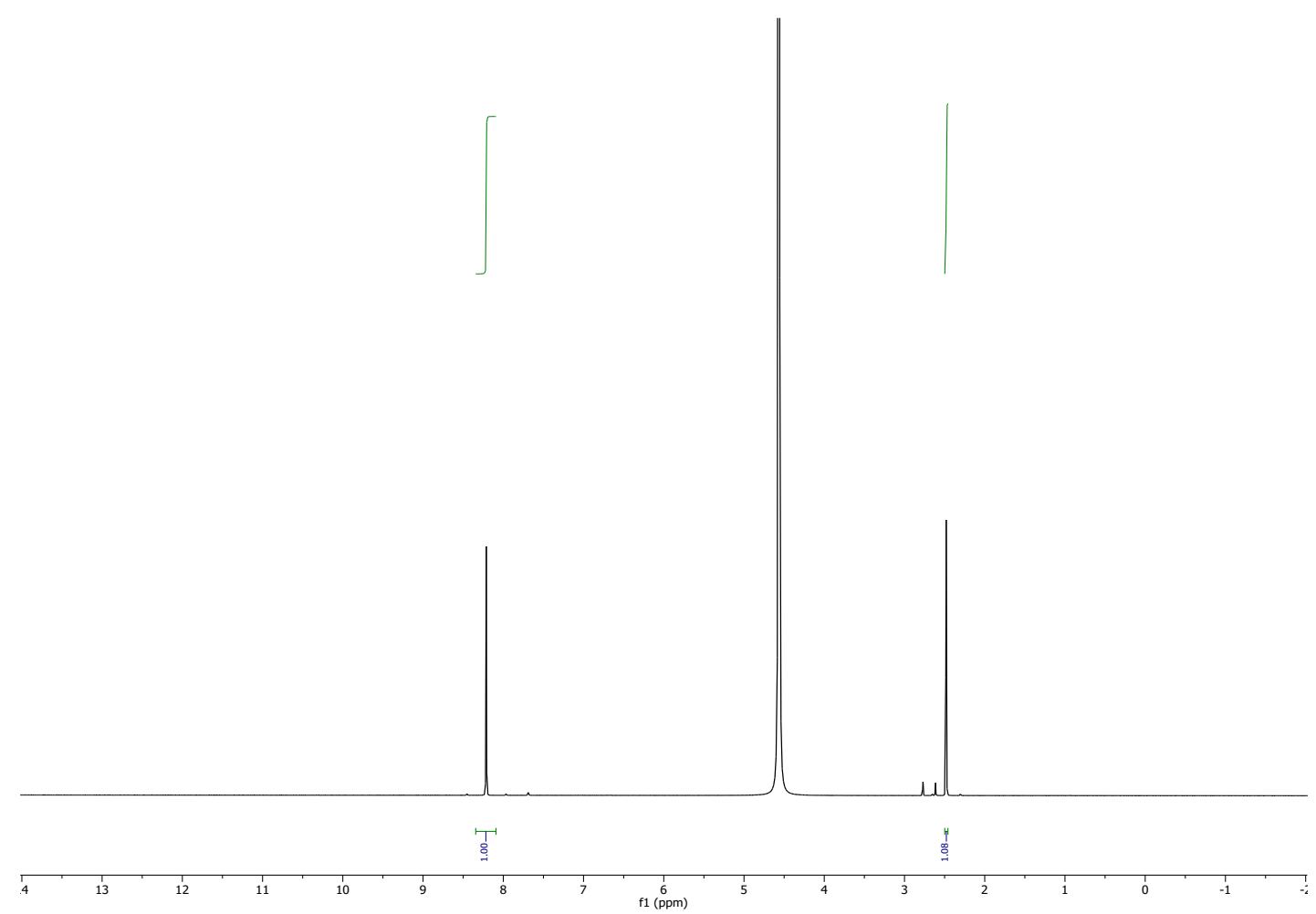

Figure S11. NMR data belonging to table 3, entry 3.

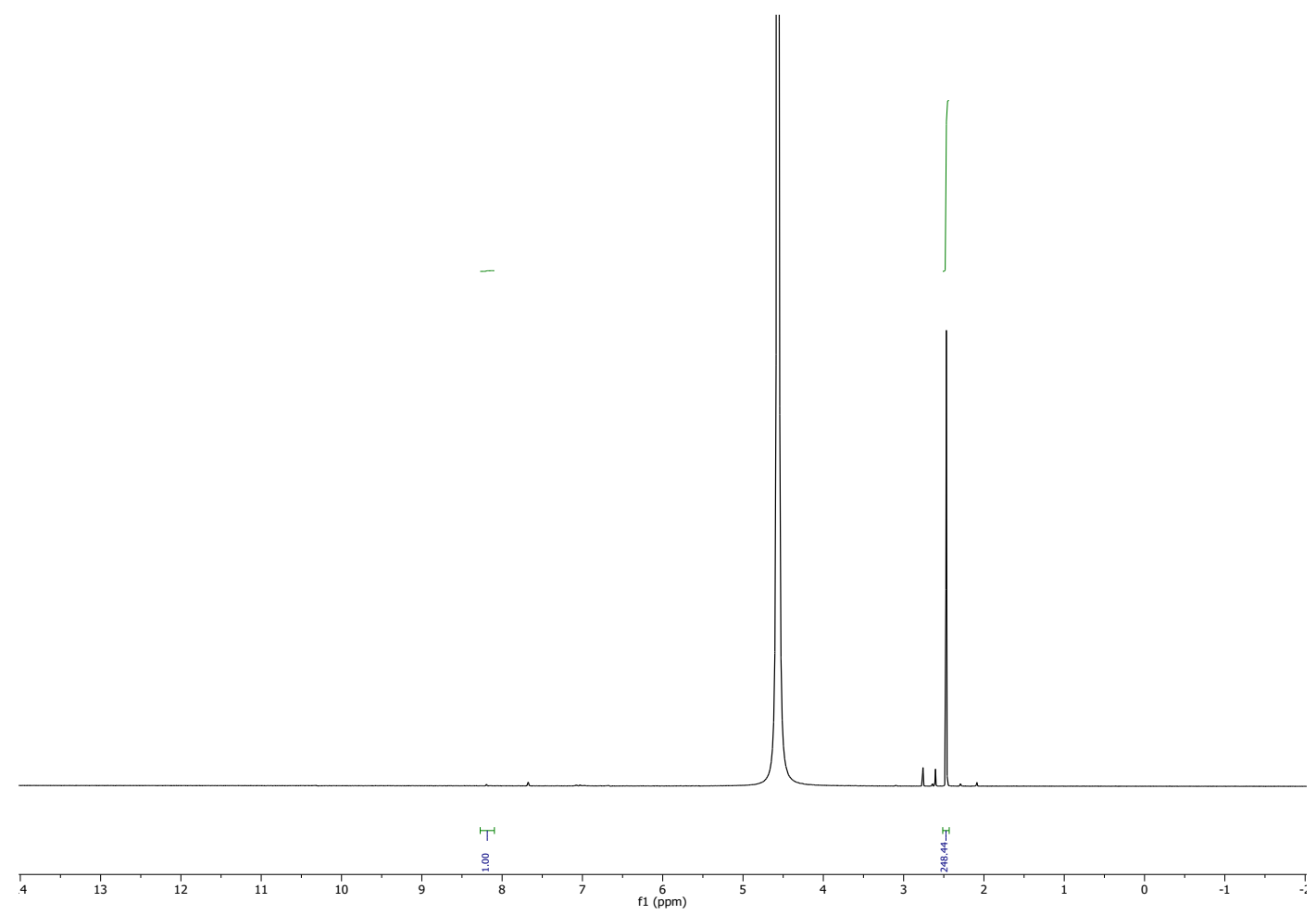

Figure S12. NMR data belonging to table 3, entry 4. 


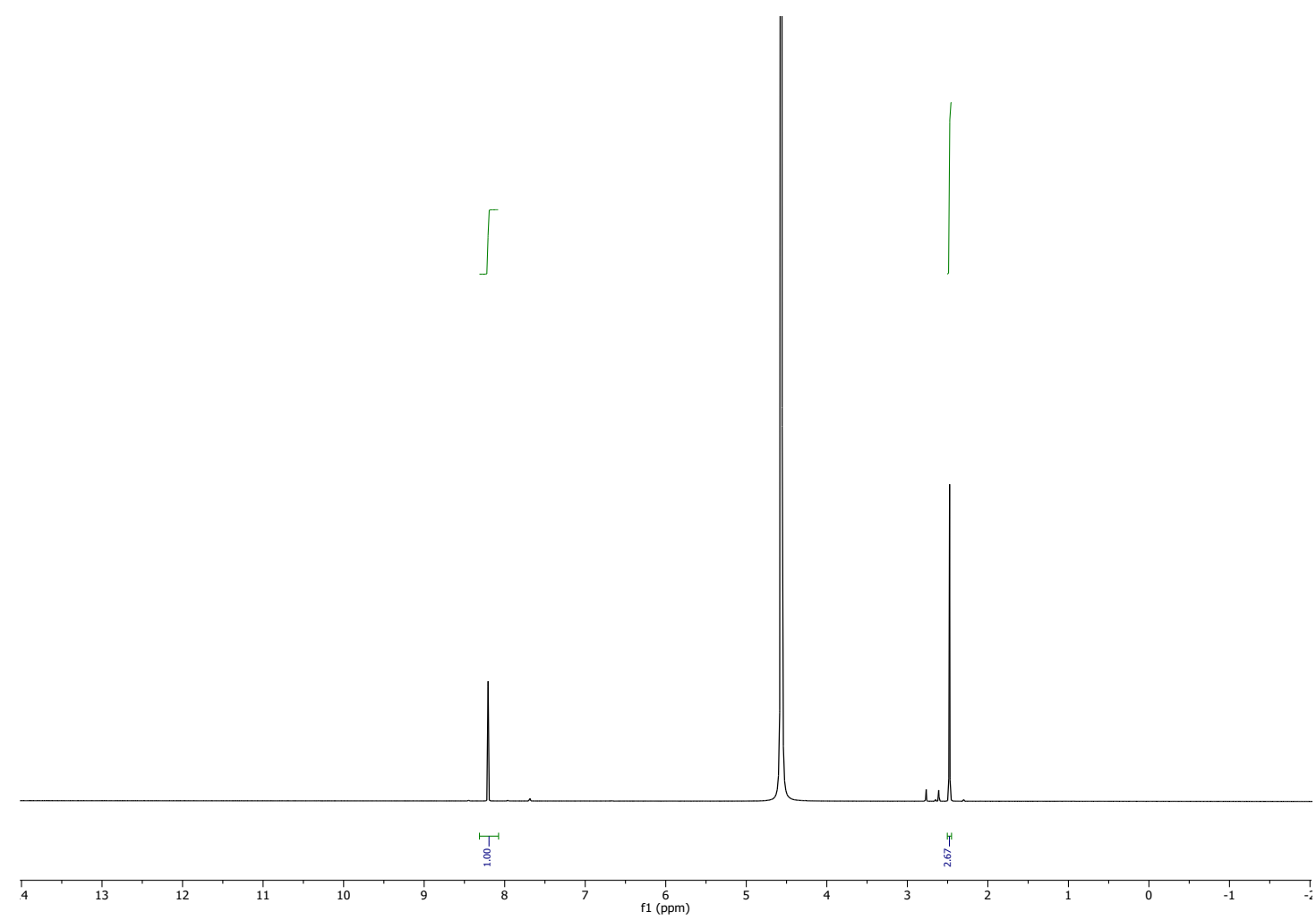

Figure S13. NMR data belonging to table 3, entry 5.

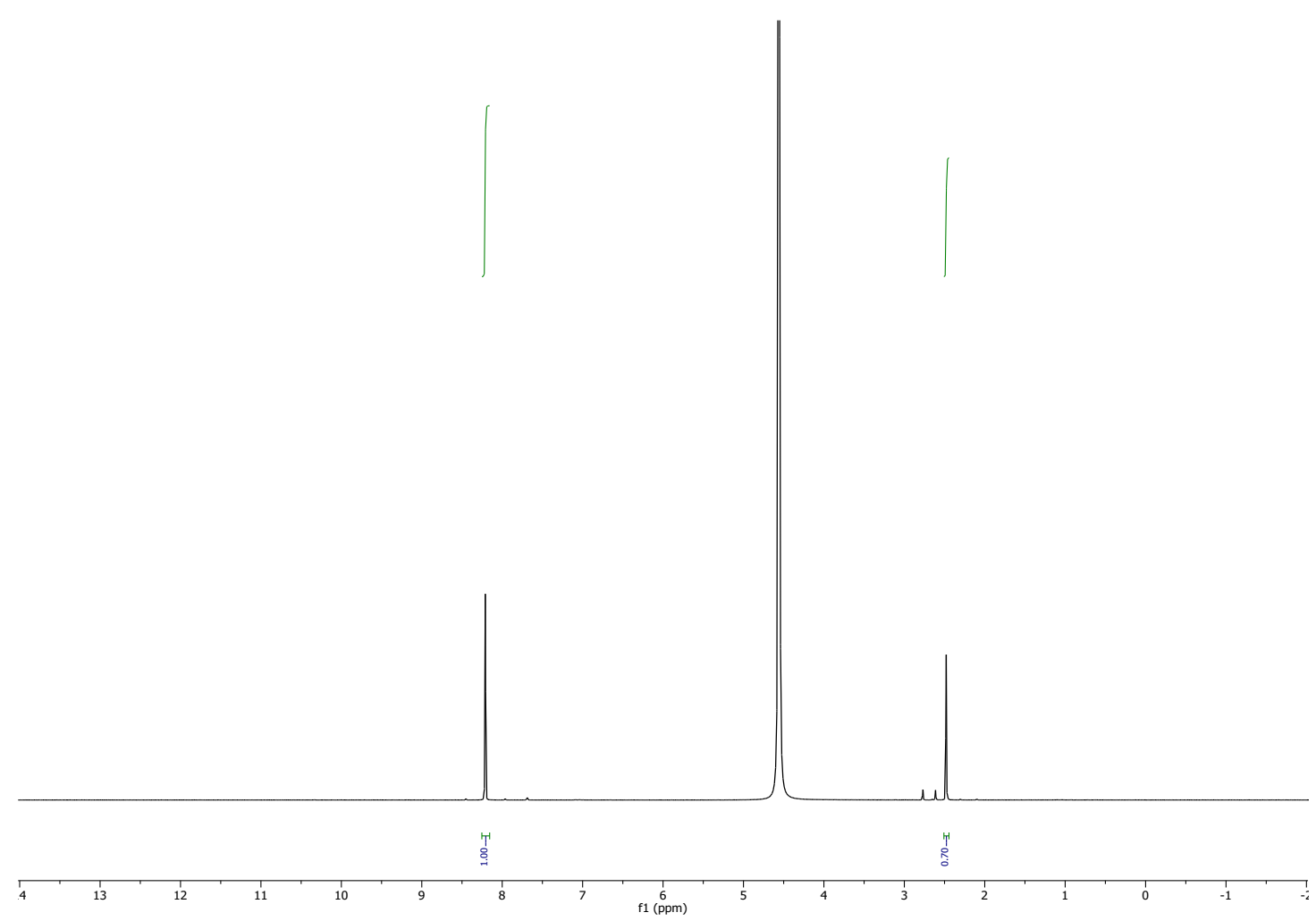

Figure S14. NMR data belonging to table 3, entry 6. 
Table S3. ${ }^{1} \mathrm{H}$ NMR peak data belonging to table 3 (main text) and Figures S9-S14.

\begin{tabular}{|l|l|}
\hline Entry & Ratio of DMSO to formate integral \\
\hline 1 & 56.95 \\
\hline 2 & 52.94 \\
\hline 3 & 1.08 \\
\hline 4 & 248.44 \\
\hline 5 & 2.67 \\
\hline 6 & 0.70 \\
\hline
\end{tabular}


Table S4. Data accompanying Figure 2 (main text), with $\mathrm{KHCO}_{3}$ as the substrate, for the $2.5 \mathrm{mmol}$ data point (the other values are found in Table 3, main text and Table S3).

\begin{tabular}{|l|l|l|l|l|}
\hline Entry & $\mathrm{KHCO}_{3}(\mathbf{m m o l})$ & $\begin{array}{l}\text { Ratio of DMSO } \\
\text { to formate } \\
\text { integral }\end{array}$ & Yield (\%) & TON \\
\hline 1 & 2.5 & 2.80 & 60.3 & 25648 \\
\hline
\end{tabular}

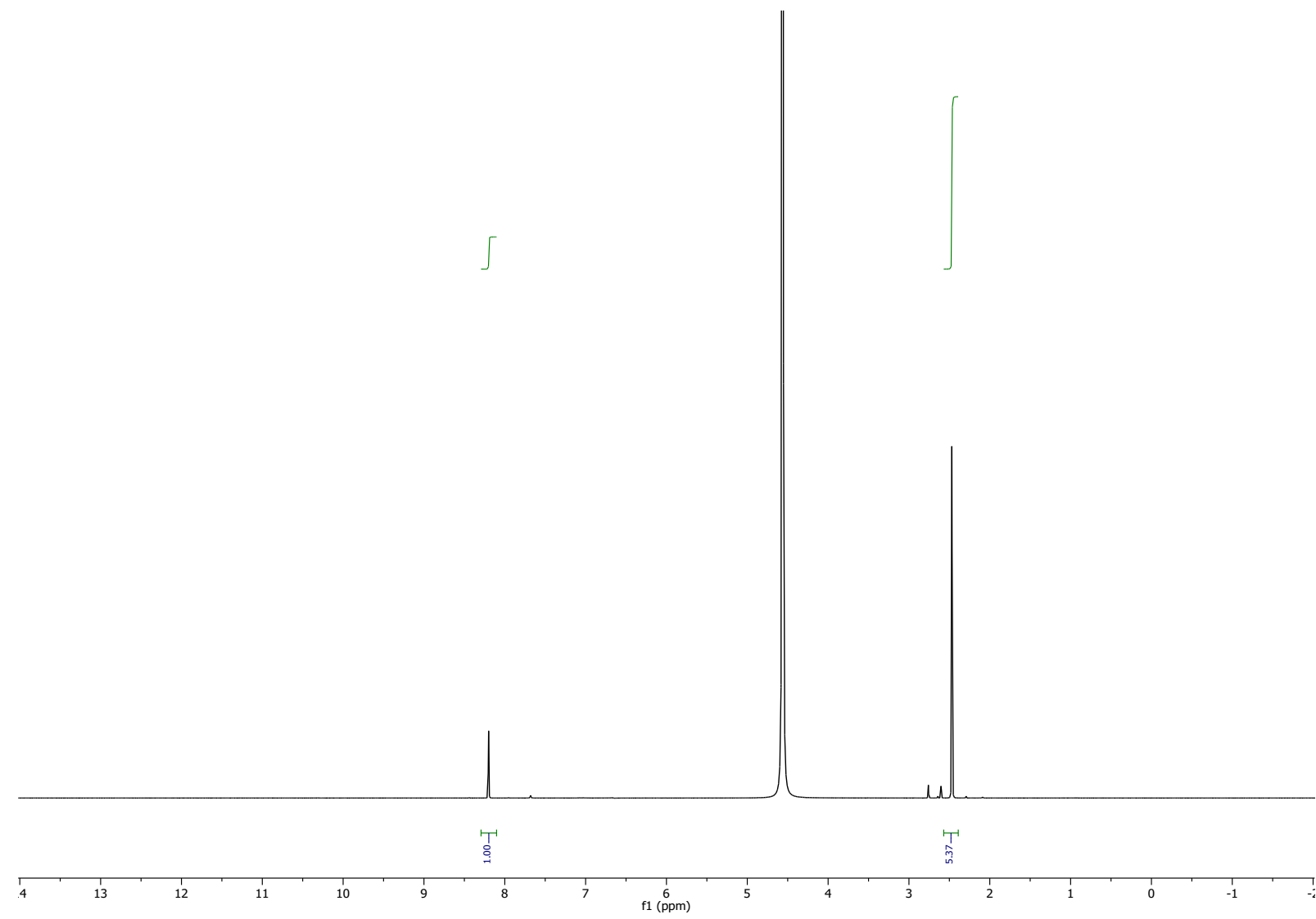

Figure S15. NMR data belonging to table S4, entry 1.

Table S5. Data accompanying Figure 2 (main text), with $\mathrm{NaHCO}_{3}$ as a substrate.

\begin{tabular}{|l|l|l|l|l|}
\hline Entry & $\mathrm{NaHCO}_{3}(\mathbf{m m o l})$ & $\begin{array}{l}\text { Ratio of DMSO } \\
\text { to formate } \\
\text { integral }\end{array}$ & Yield (\%) & TON \\
\hline 1 & 5 & 2.80 & 60.3 & 25648 \\
\hline 2 & 7.5 & 2.26 & 49.8 & 31777 \\
\hline 3 & 10 & 2.58 & 32.7 & 27835 \\
\hline
\end{tabular}




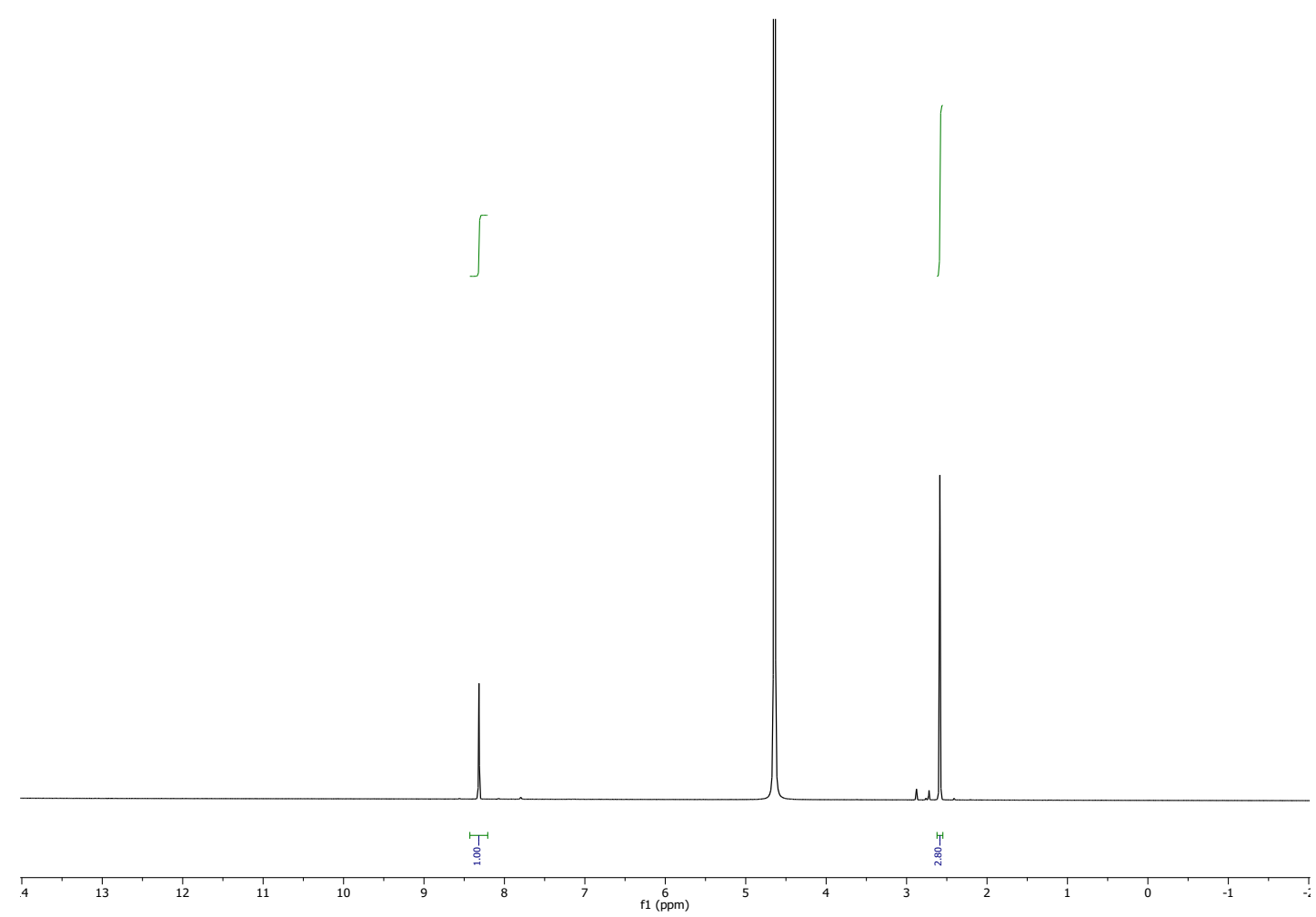

Figure S16. NMR data belonging to table S5, entry 1.

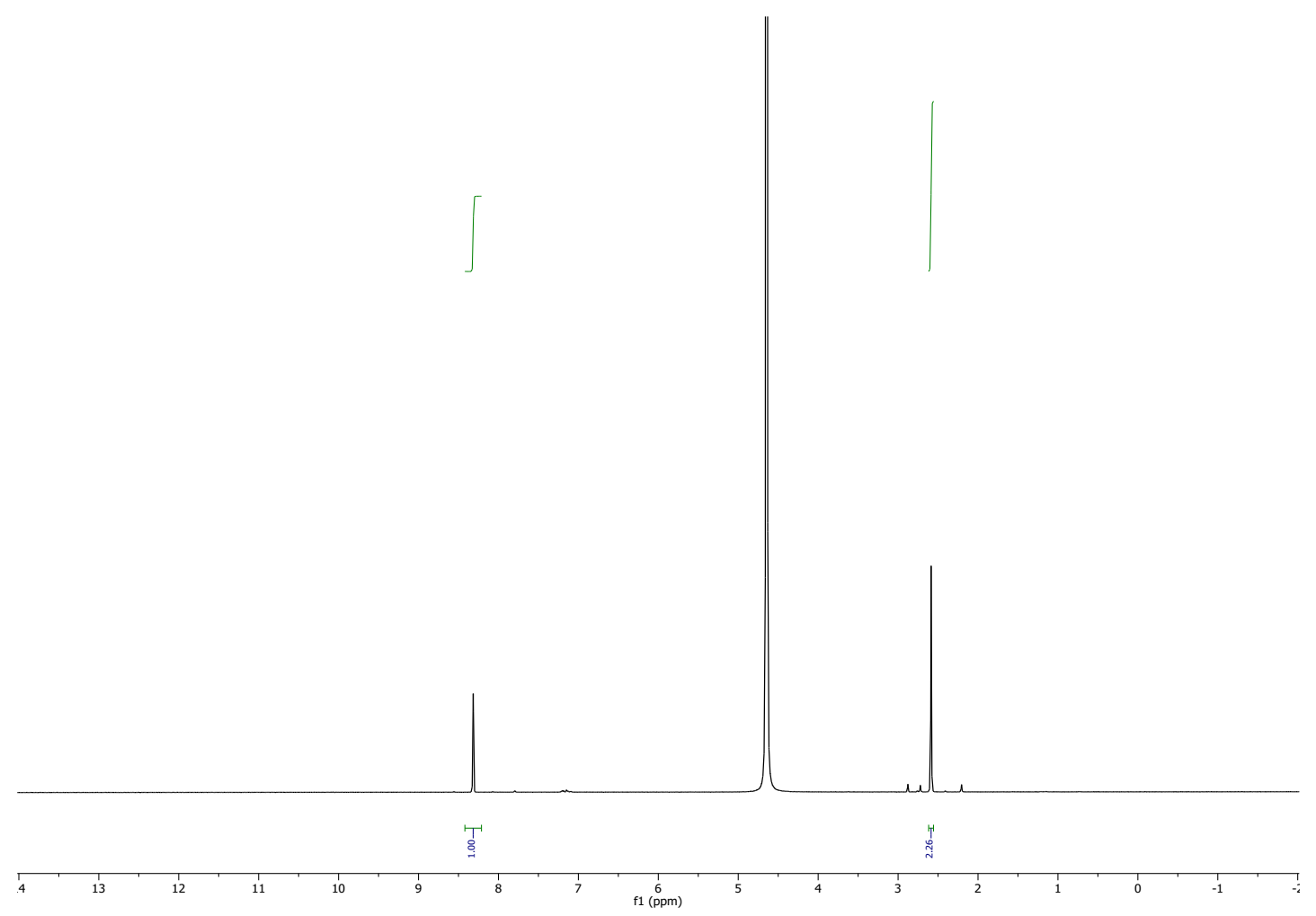

Figure S17. NMR data belonging to table S5, entry 2. 


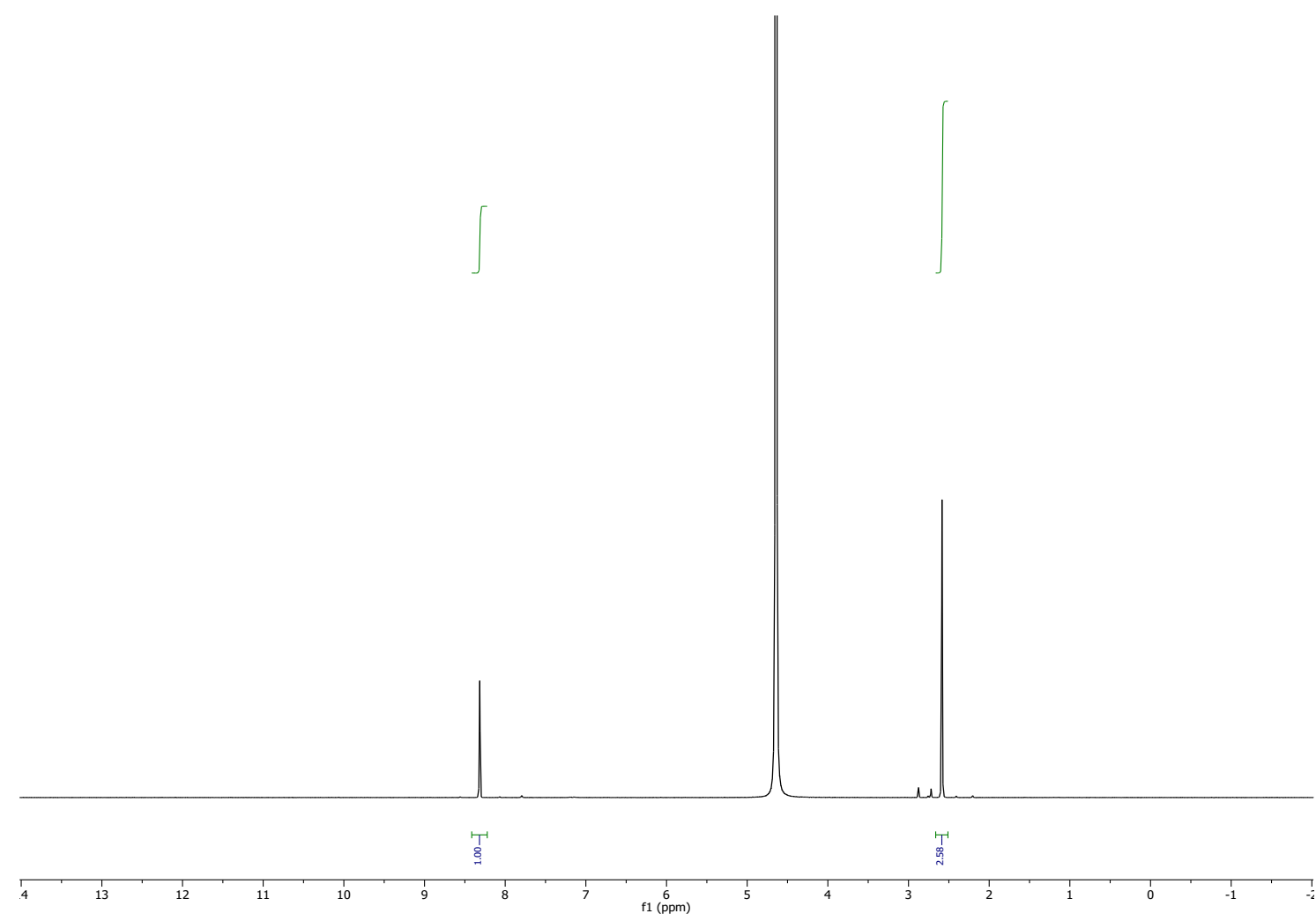

Figure S18. NMR data belonging to table S5, entry 3. 
Table S6. Data accompanying Figure 3 (main text), for $\mathrm{T}=65^{\circ}$.

\begin{tabular}{|l|l|l|l|l|}
\hline Entry & Pressure (bar) & $\begin{array}{l}\text { Ratio of DMSO to } \\
\text { formate integral }\end{array}$ & Yield (\%) & TON \\
\hline 1 & 5 & 5.02 & 33.7 & 14306 \\
\hline 2 & 10 & 3.37 & 50.2 & 21310 \\
\hline 3 & 20 & 2.70 & 62.6 & 26598 \\
\hline 4 & 40 & 2.31 & 73.2 & 31089 \\
\hline 5 & 60 & 2.29 & 73.9 & 31360 \\
\hline
\end{tabular}

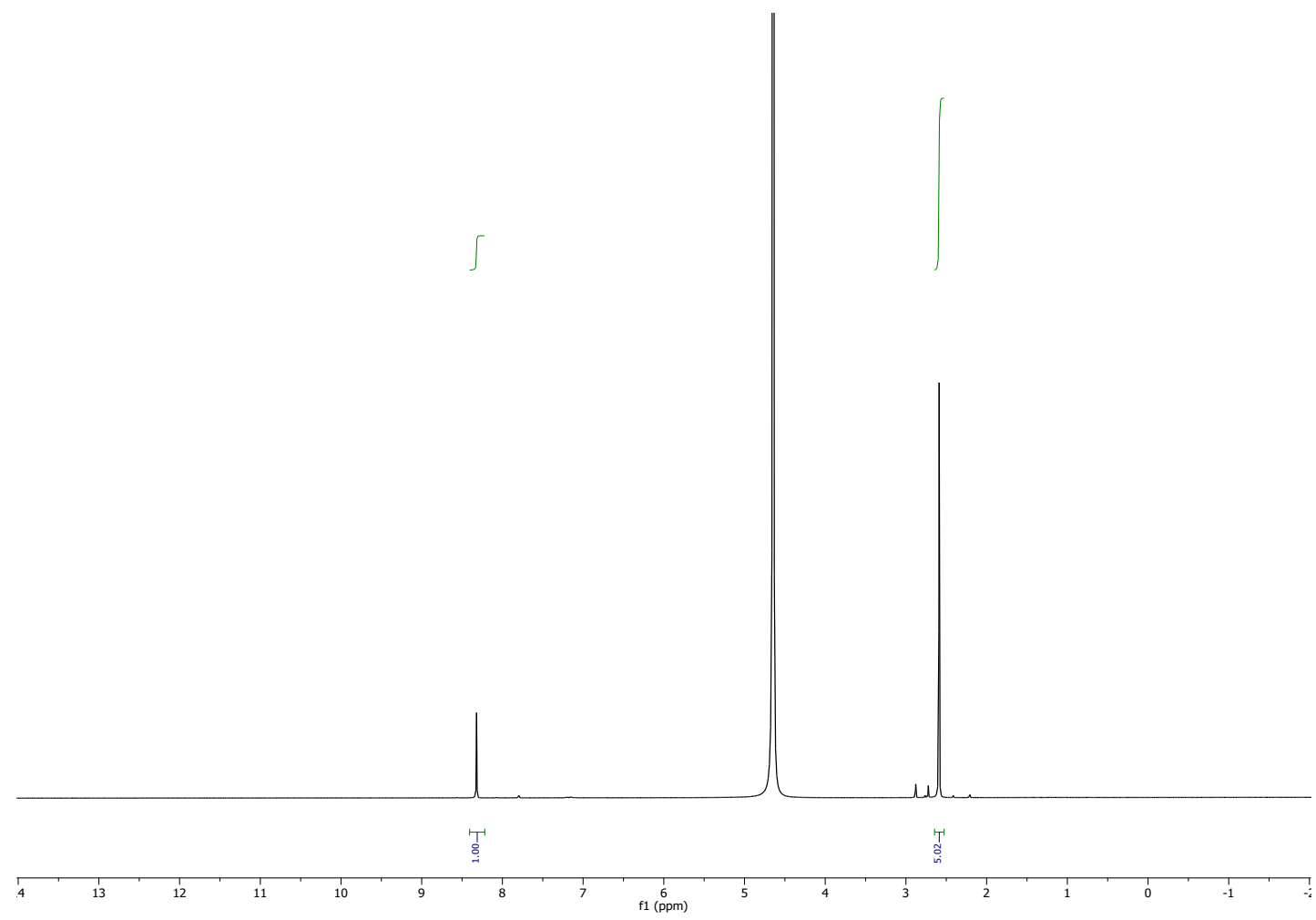

Figure S19. NMR data belonging to table S6, entry 1. 


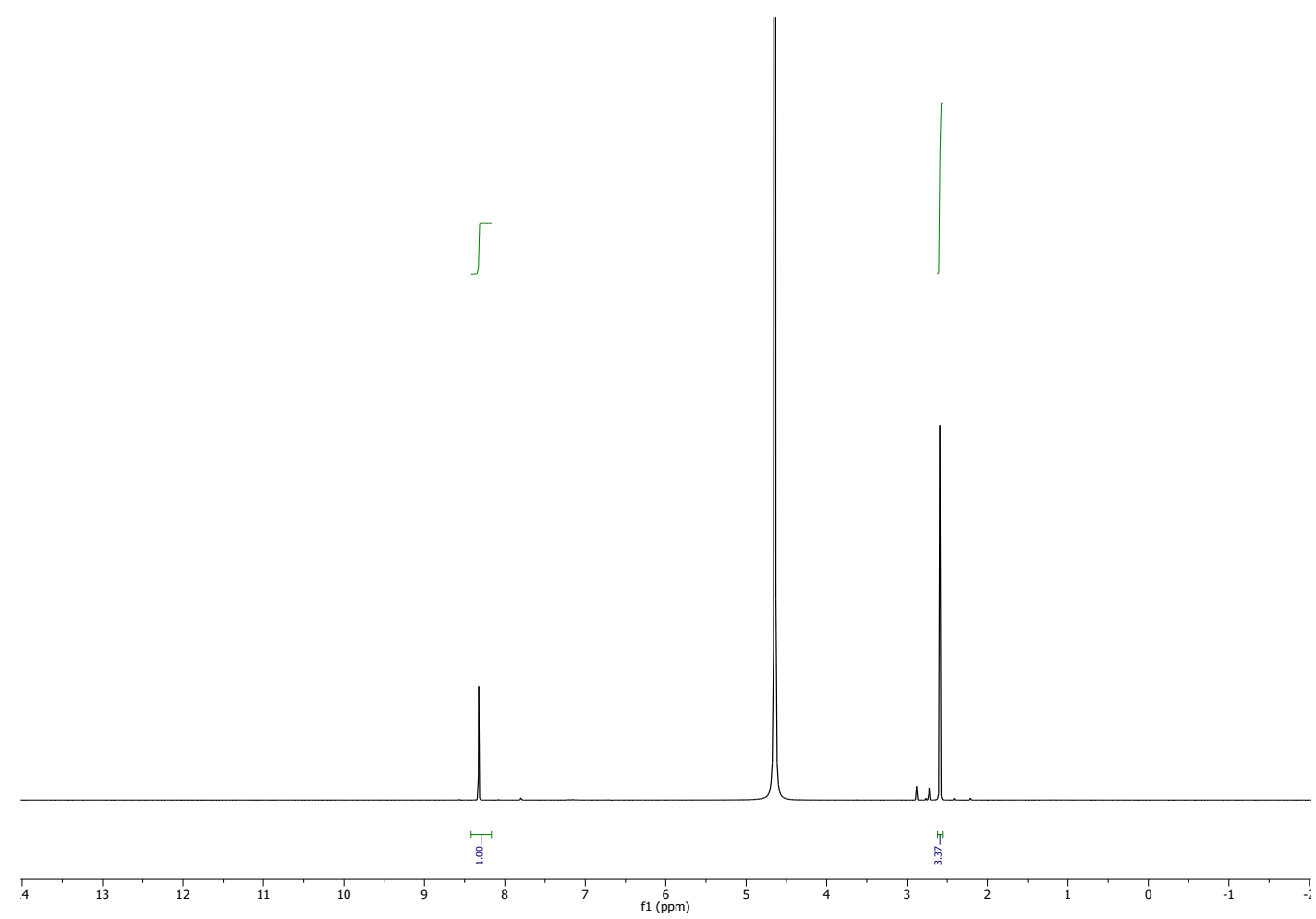

Figure S20. NMR data belonging to table S6, entry 2.

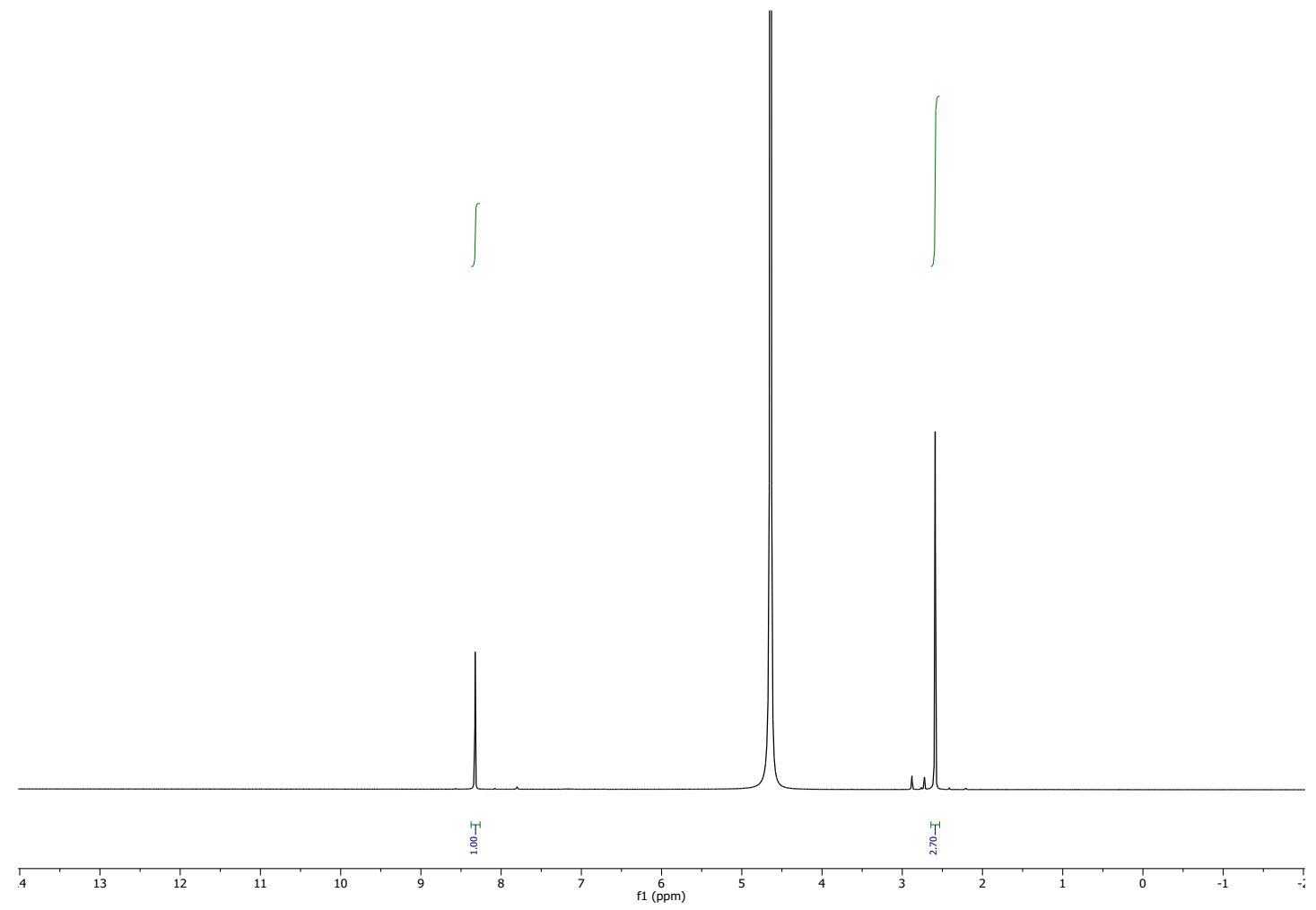

Figure S21. NMR data belonging to table S6, entry 3. 


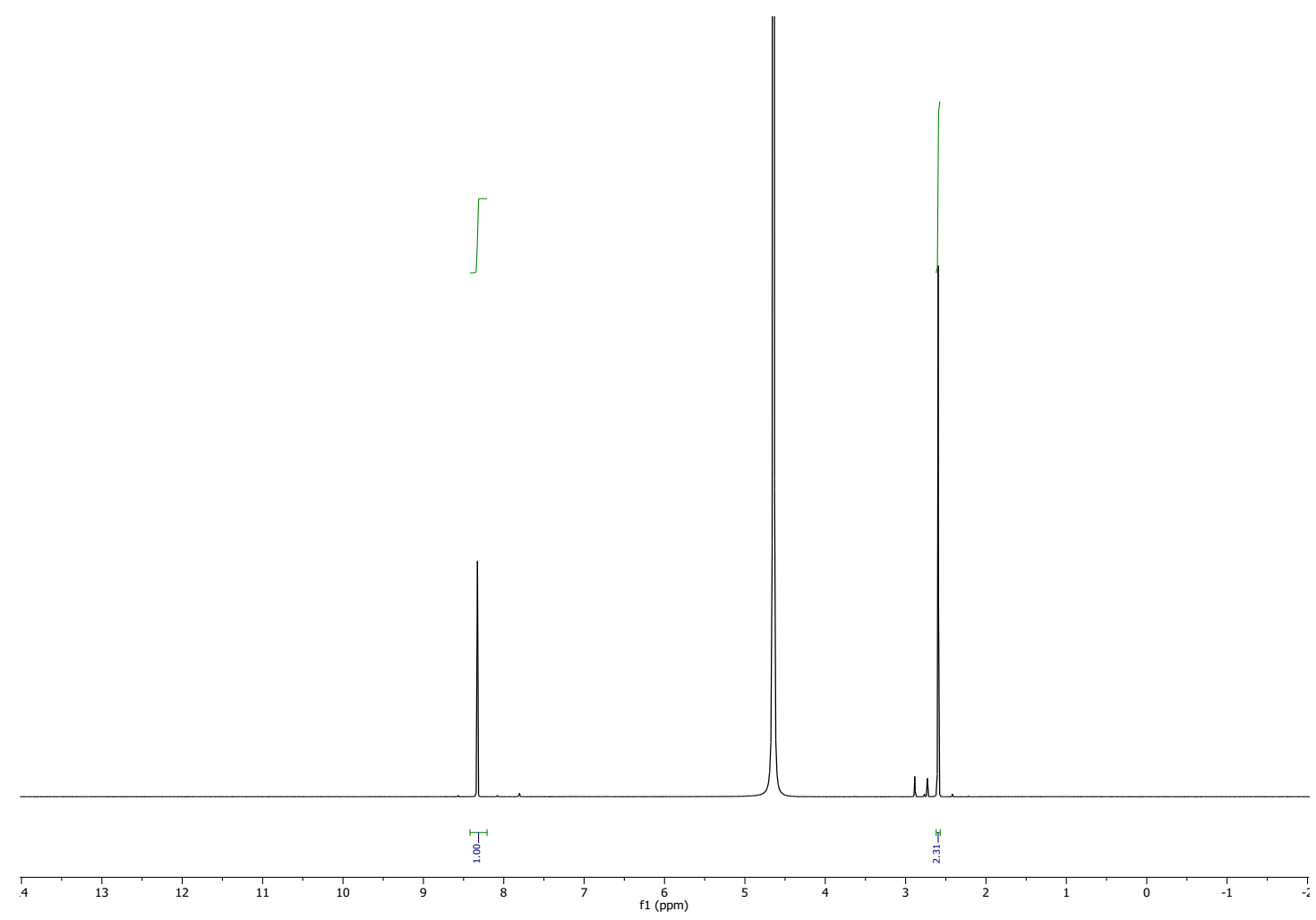

Figure S22. NMR data belonging to table S6, entry 4.

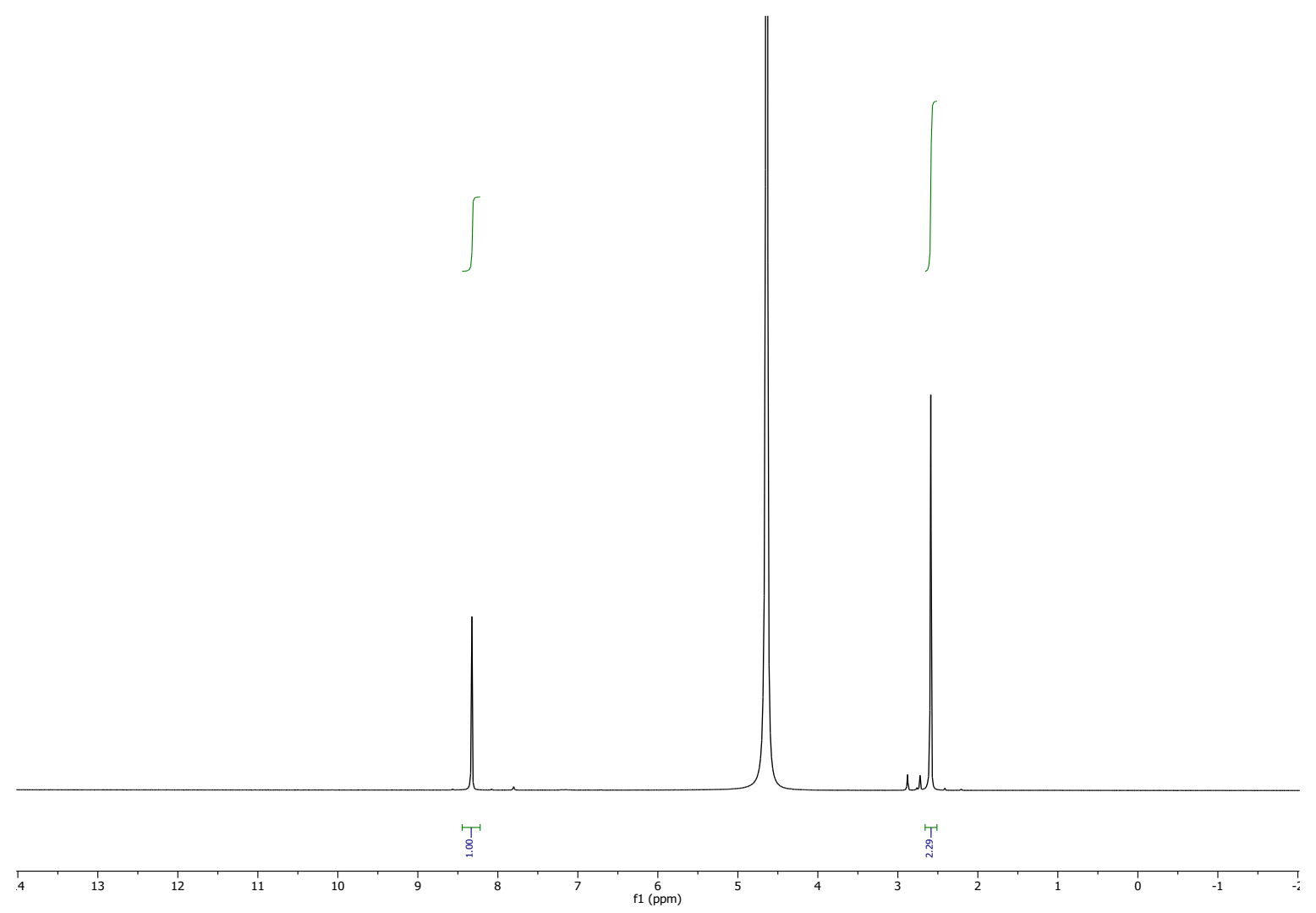

Figure S23. NMR data belonging to table S6, entry 5. 
Table S7. Data accompanying Figure 3 (main text), for $\mathrm{T}=90^{\circ}$.

\begin{tabular}{|l|l|l|l|l|}
\hline Entry & Pressure (bar) & $\begin{array}{l}\text { Ratio of DMSO } \\
\text { to formate } \\
\text { integral }\end{array}$ & Yield (\%) & TON \\
\hline 1 & 5 & 4.86 & 35.3 & 14777 \\
\hline 2 & 10 & 4.80 & 35.5 & 14961 \\
\hline 3 & 20 & 2.84 & 60.1 & 25287 \\
\hline 4 & 40 & 2.32 & 73.4 & 30955 \\
\hline 5 & 50 & 1.89 & 89.1 & 37997 \\
\hline
\end{tabular}

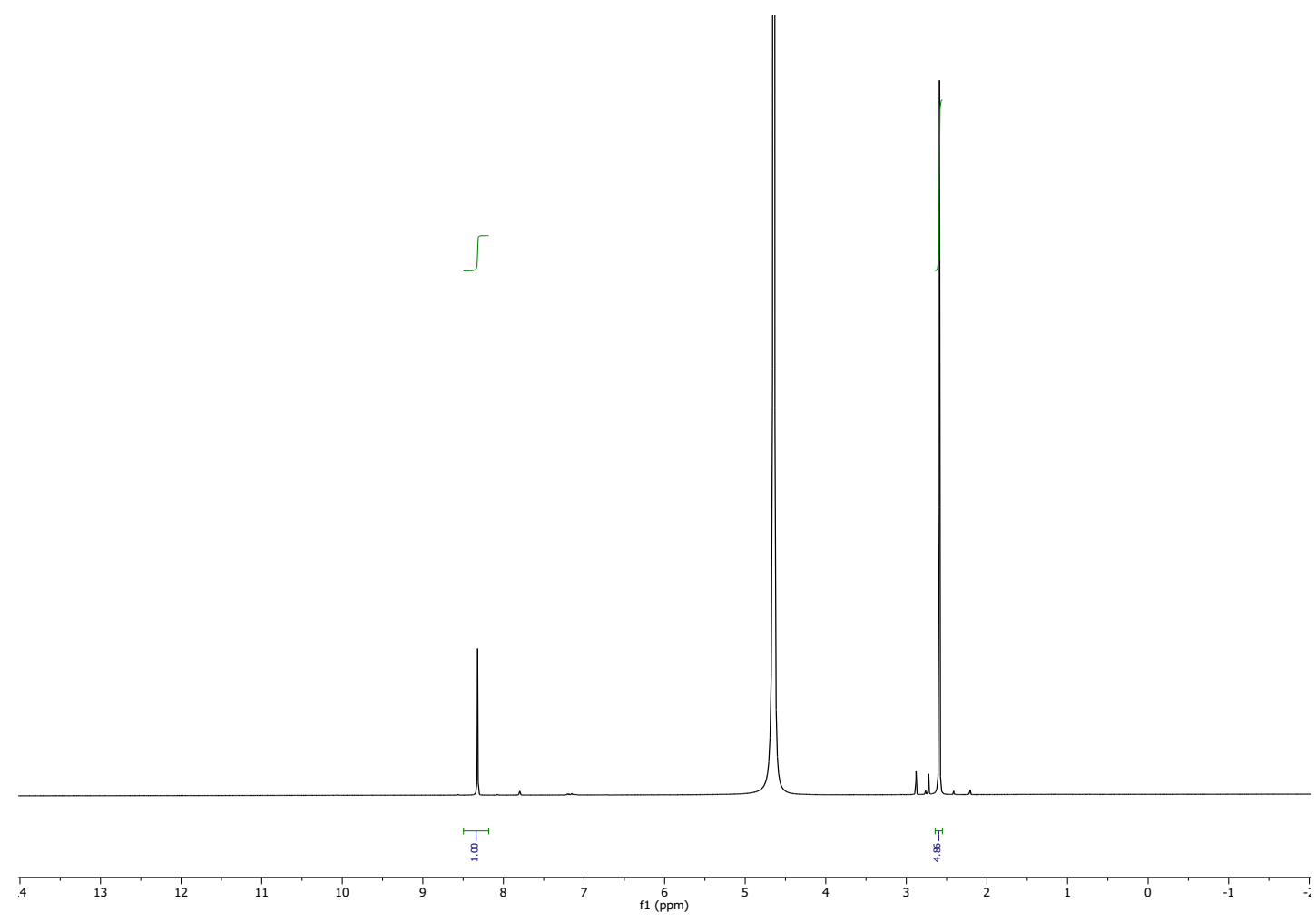

Figure S24. NMR data belonging to table S7, entry 1. 


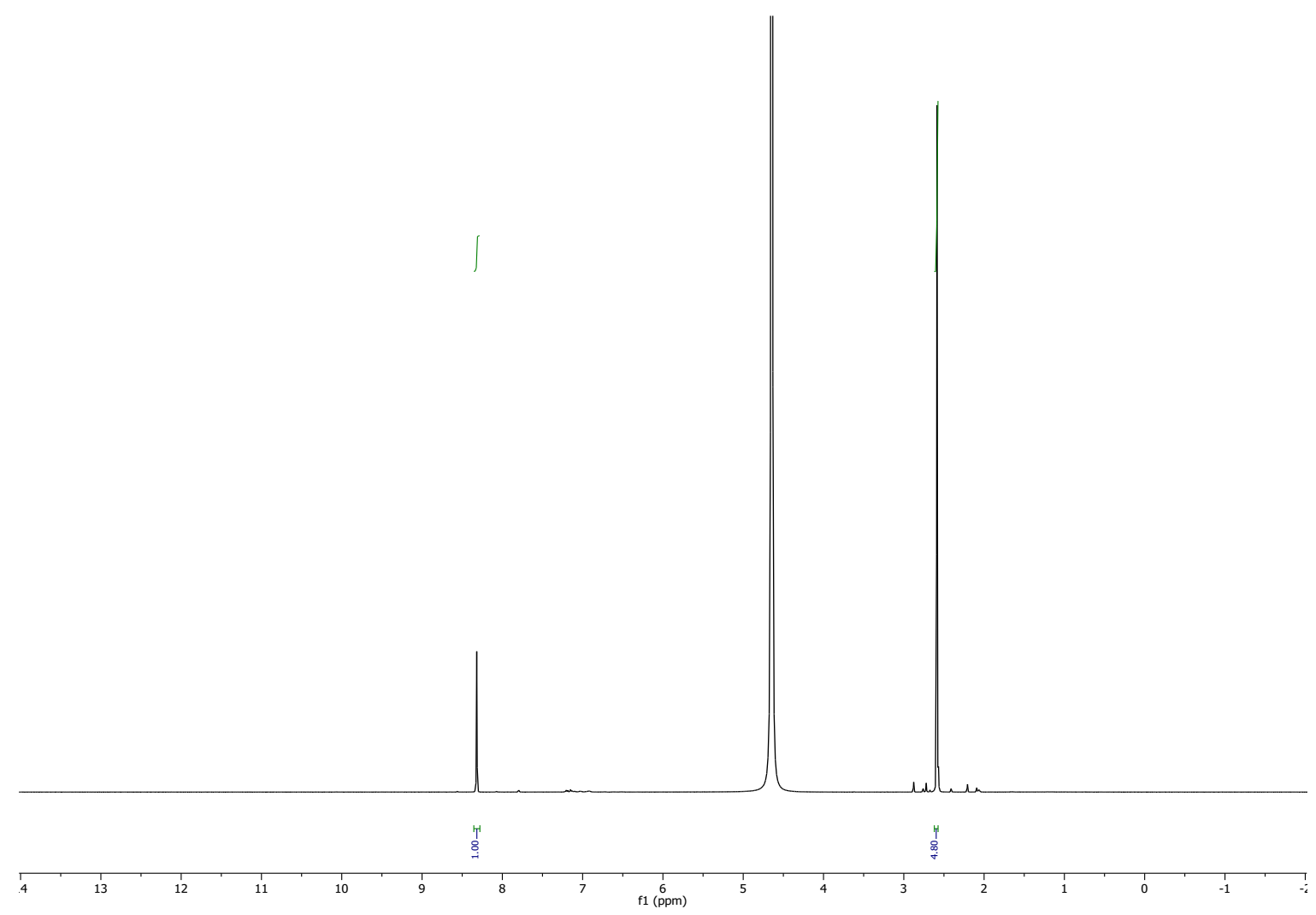

Figure S25. NMR data belonging to table S7, entry 2.

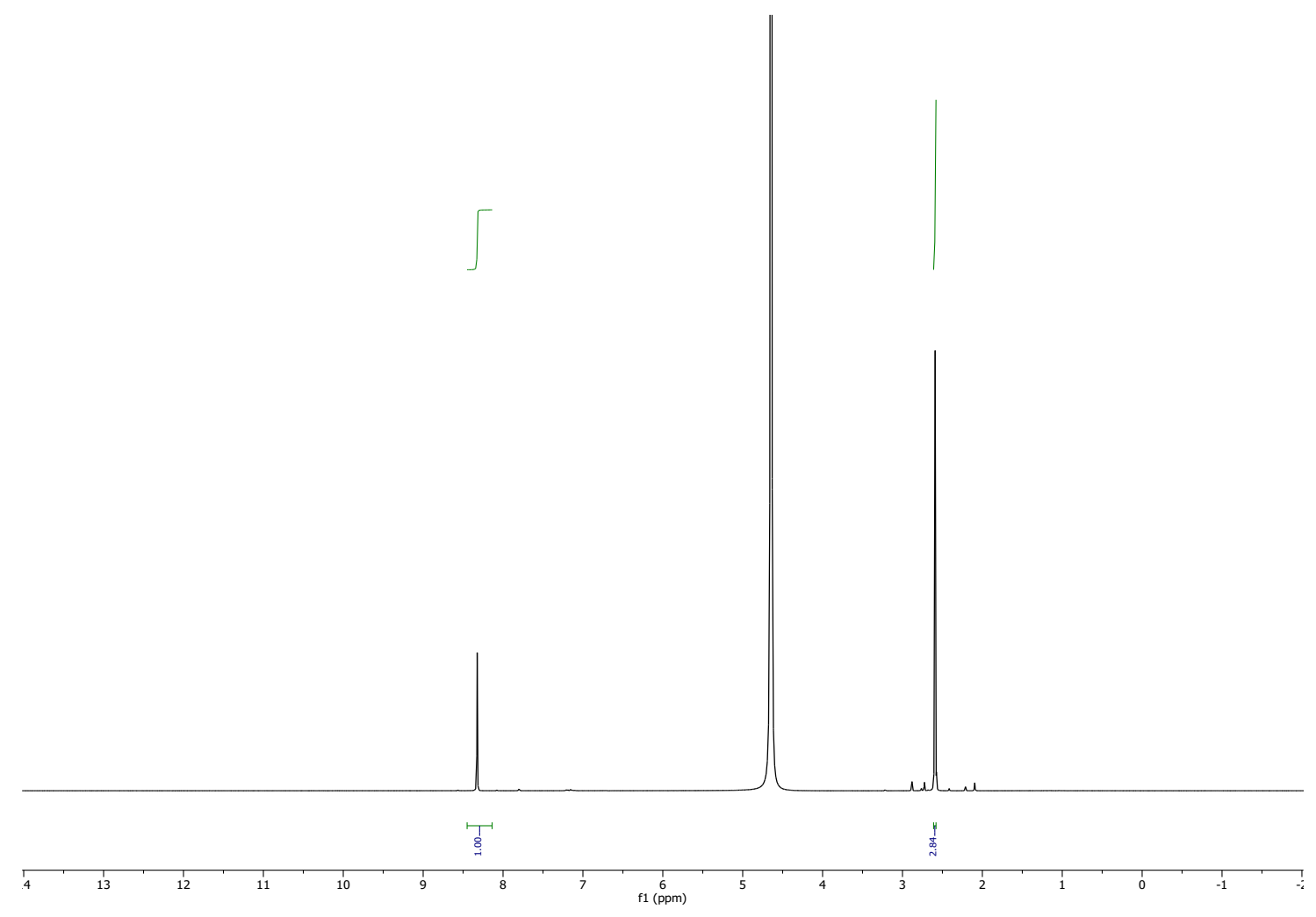

Figure S26. NMR data belonging to table S7, entry 3. 


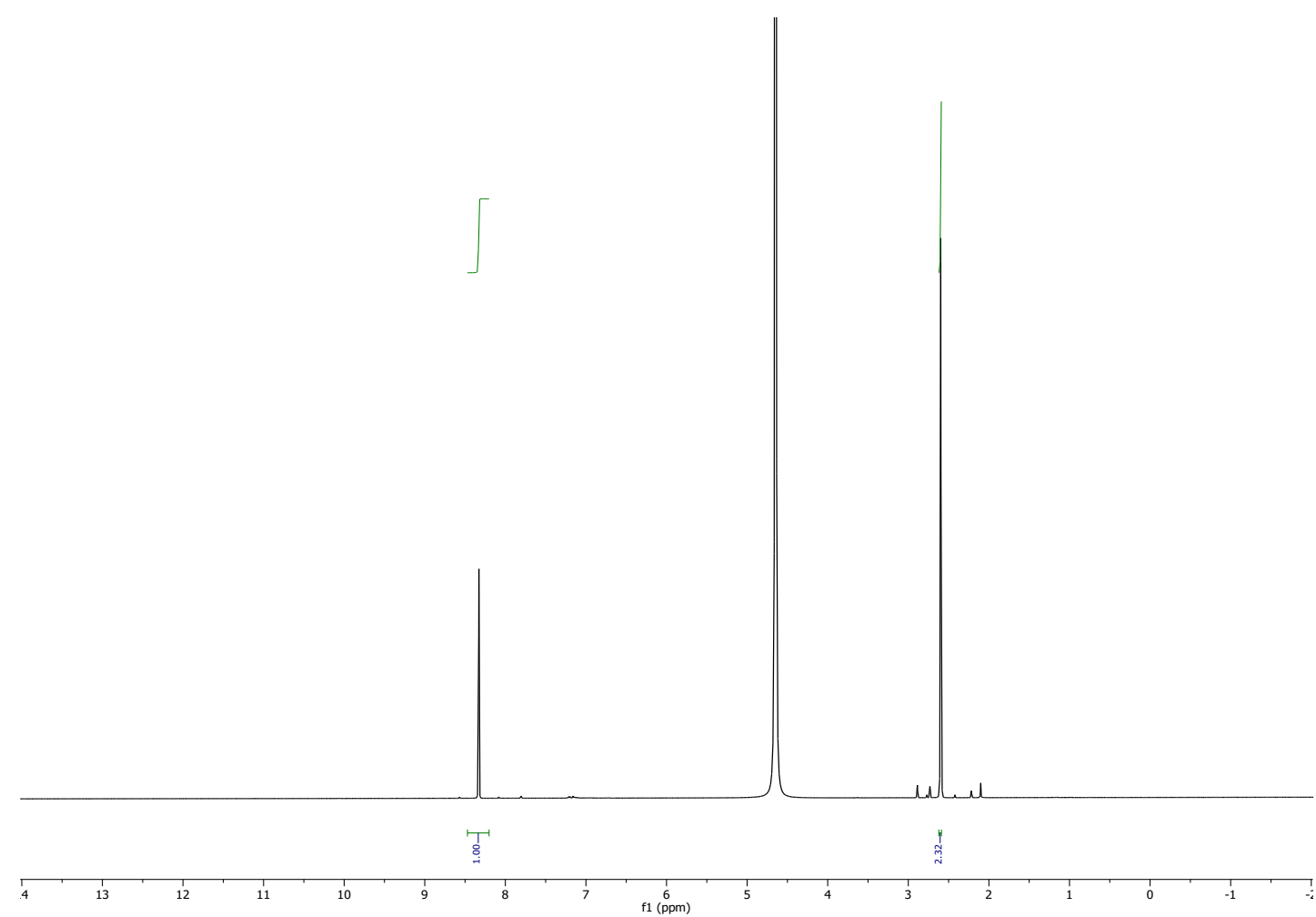

Figure S27. NMR data belonging to table S7, entry 4.

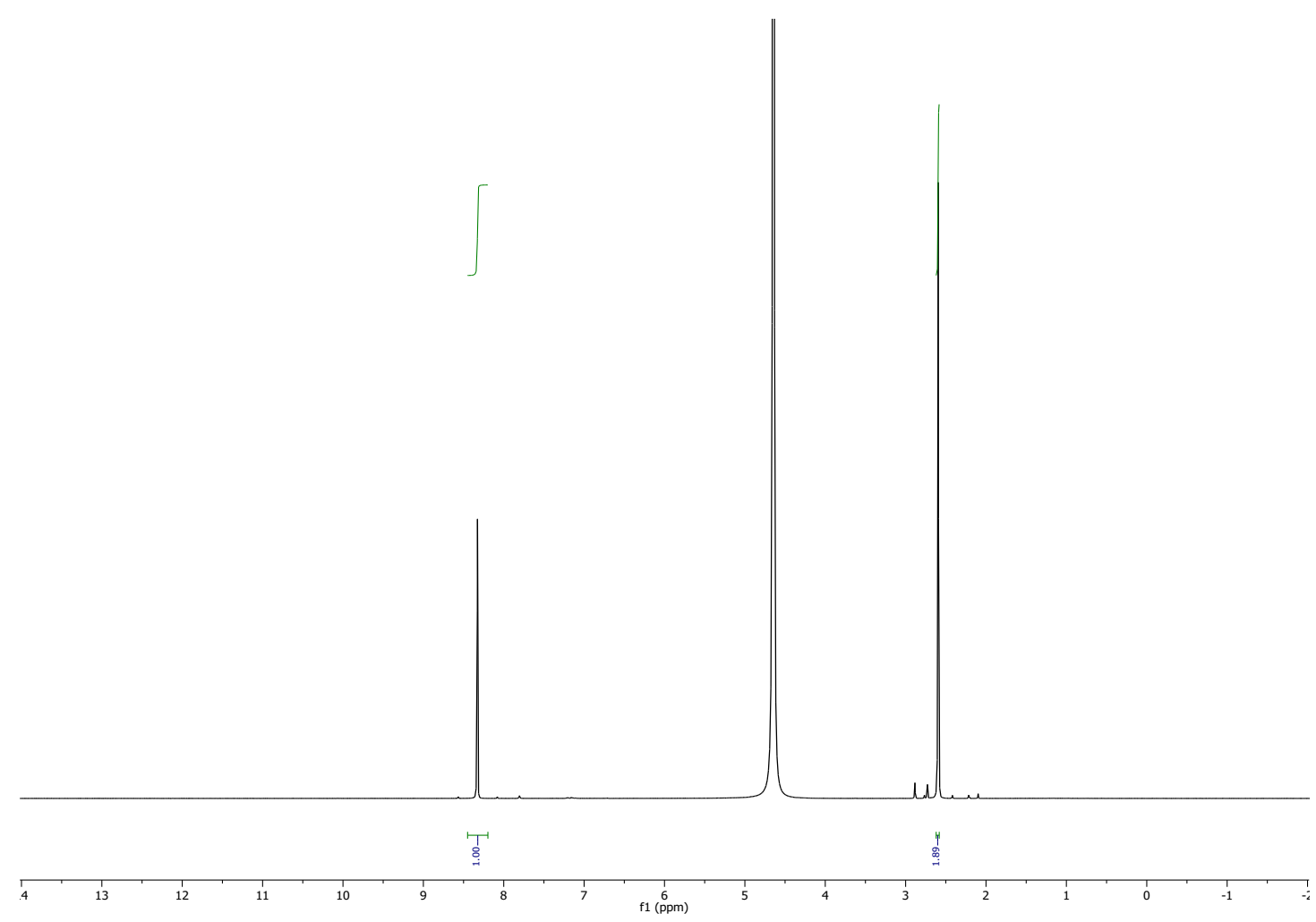

Figure S28. NMR data belonging to table S7, entry 5. 
Table S8. Data accompanying Figure 3 (main text), for $\mathrm{T}=120^{\circ}$.

\begin{tabular}{|l|l|l|l|l|}
\hline Entry & Pressure (bar) & $\begin{array}{l}\text { Ratio of DMSO } \\
\text { to formate } \\
\text { integral }\end{array}$ & Yield (\%) & TON \\
\hline 1 & 5 & 199.01 & 0.8 & 361 \\
\hline 2 & 10 & 9.00 & 18.8 & 7979 \\
\hline 3 & 20 & 5.00 & 33.8 & 14363 \\
\hline 4 & 40 & 2.54 & 66.6 & 28274 \\
\hline 5 & 53 & 2.06 & 82.1 & 34862 \\
\hline
\end{tabular}

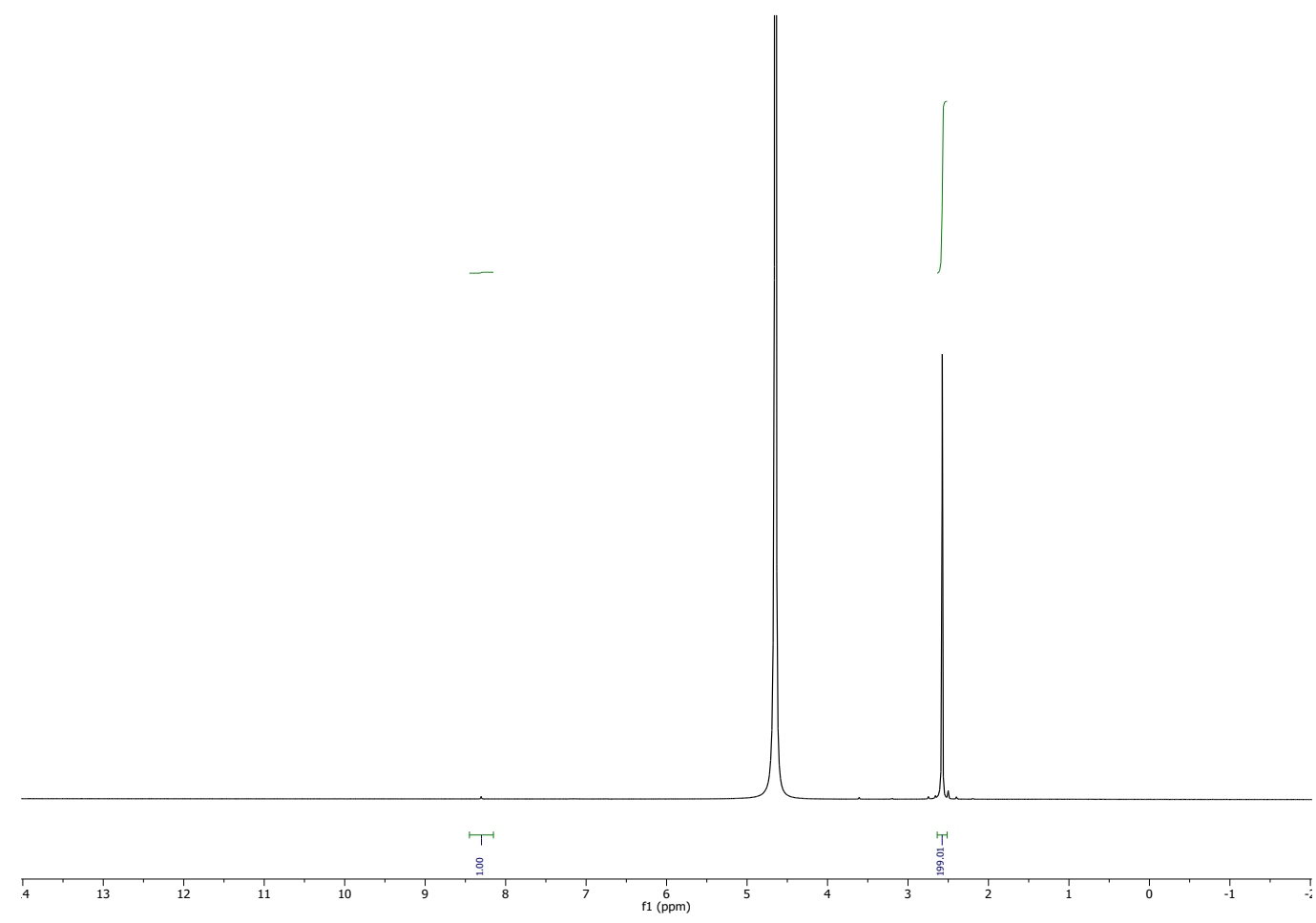

Figure S29. NMR data belonging to table S8, entry 1. 


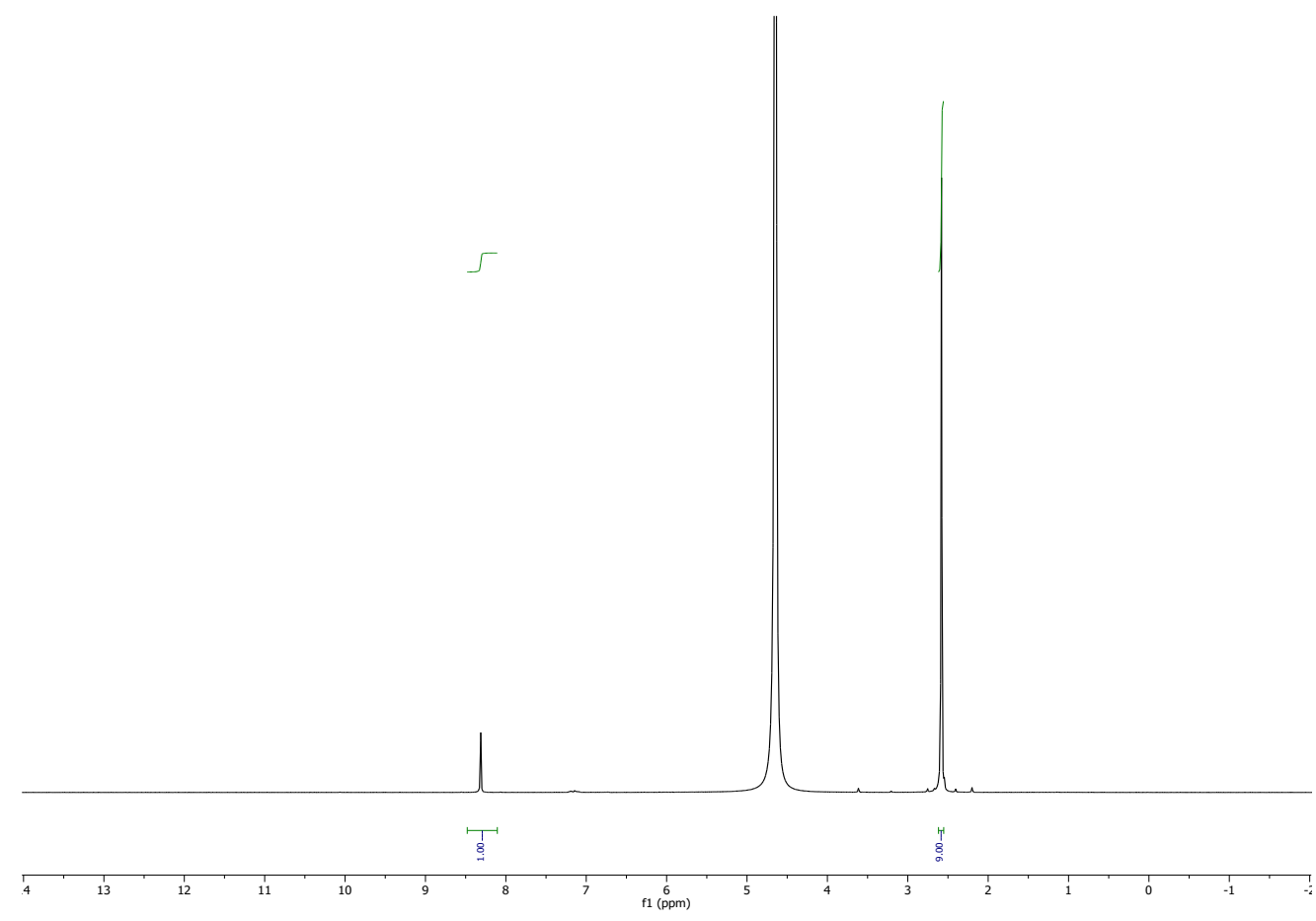

Figure S30. NMR data belonging to table S8, entry 2 .

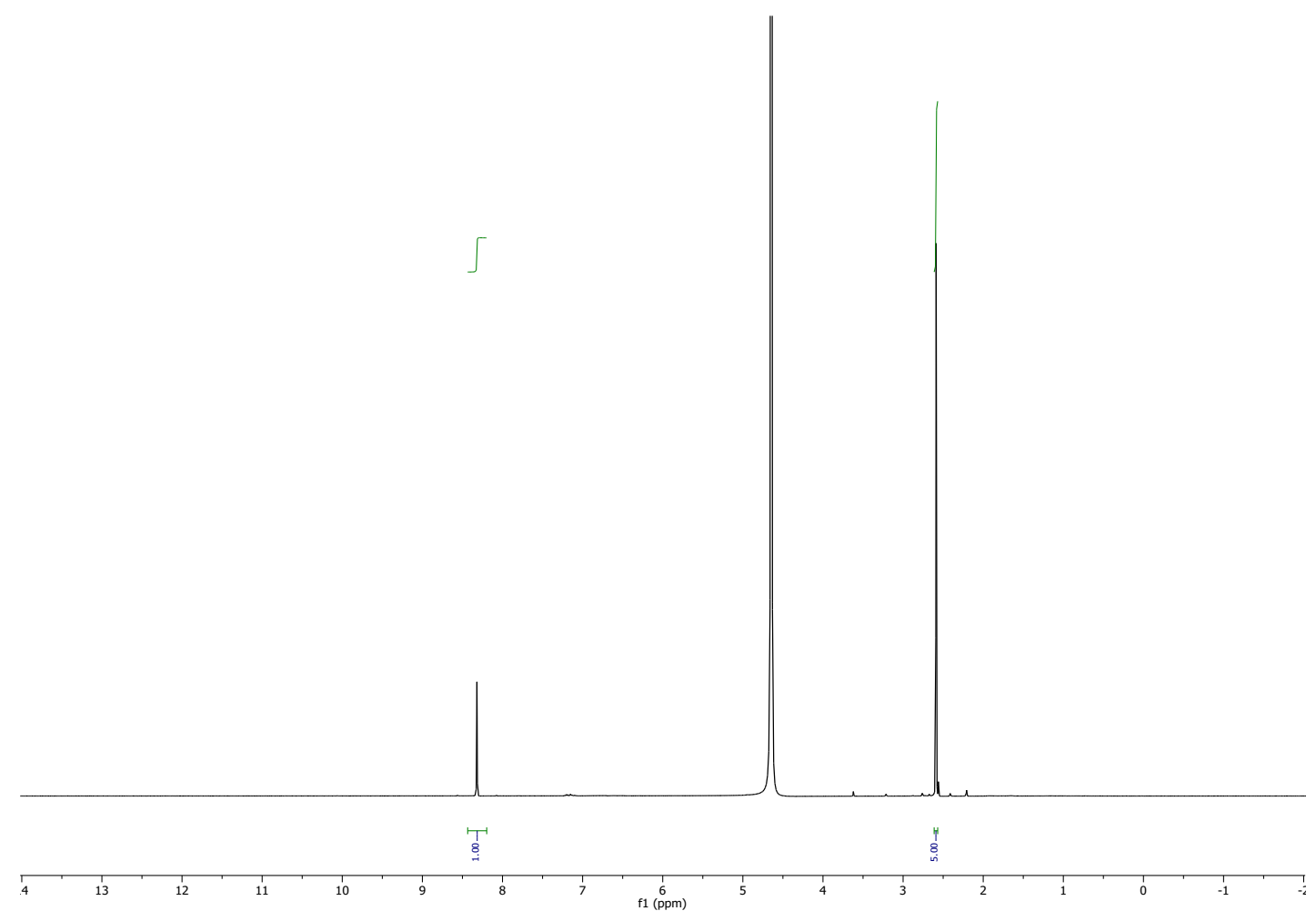

Figure S31. NMR data belonging to table S8, entry 3. 


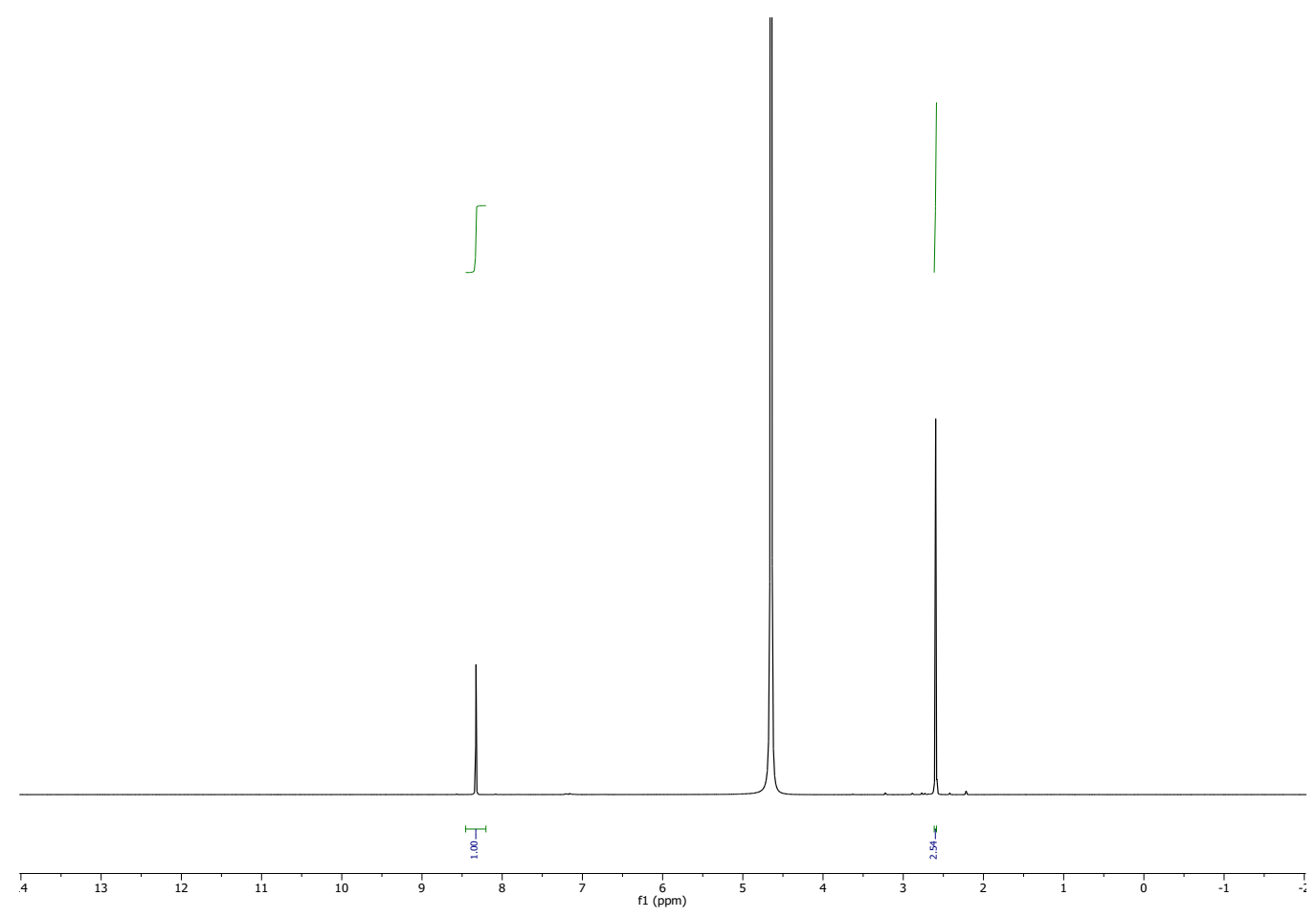

Figure S32. NMR data belonging to table S8, entry 4.

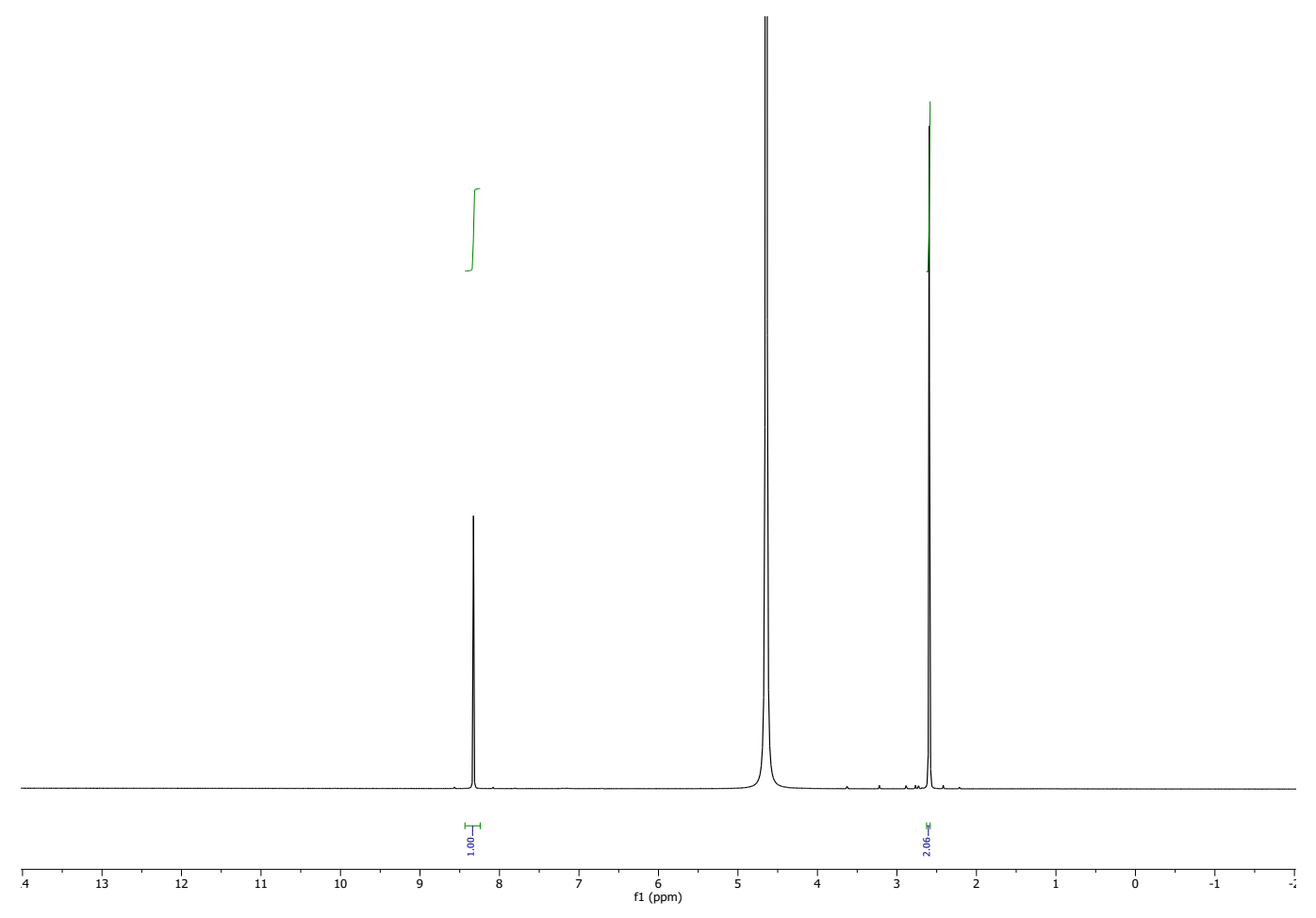

Figure S33. NMR data belonging to table S8, entry 5. 
${ }^{1}$ H NMR data accompanying data provided in Table 4

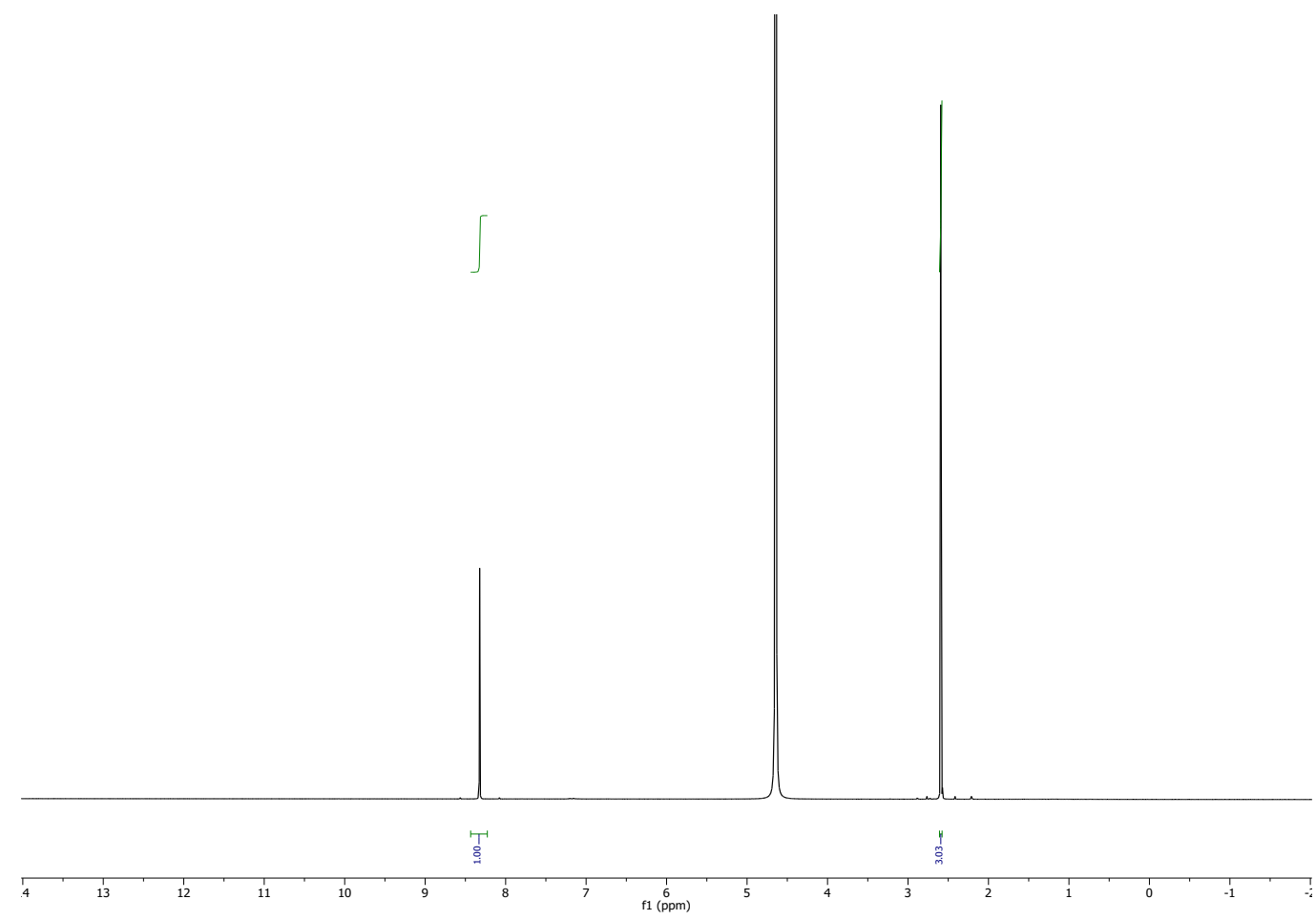

Figure S34. NMR data belonging to table S9, entry 1.

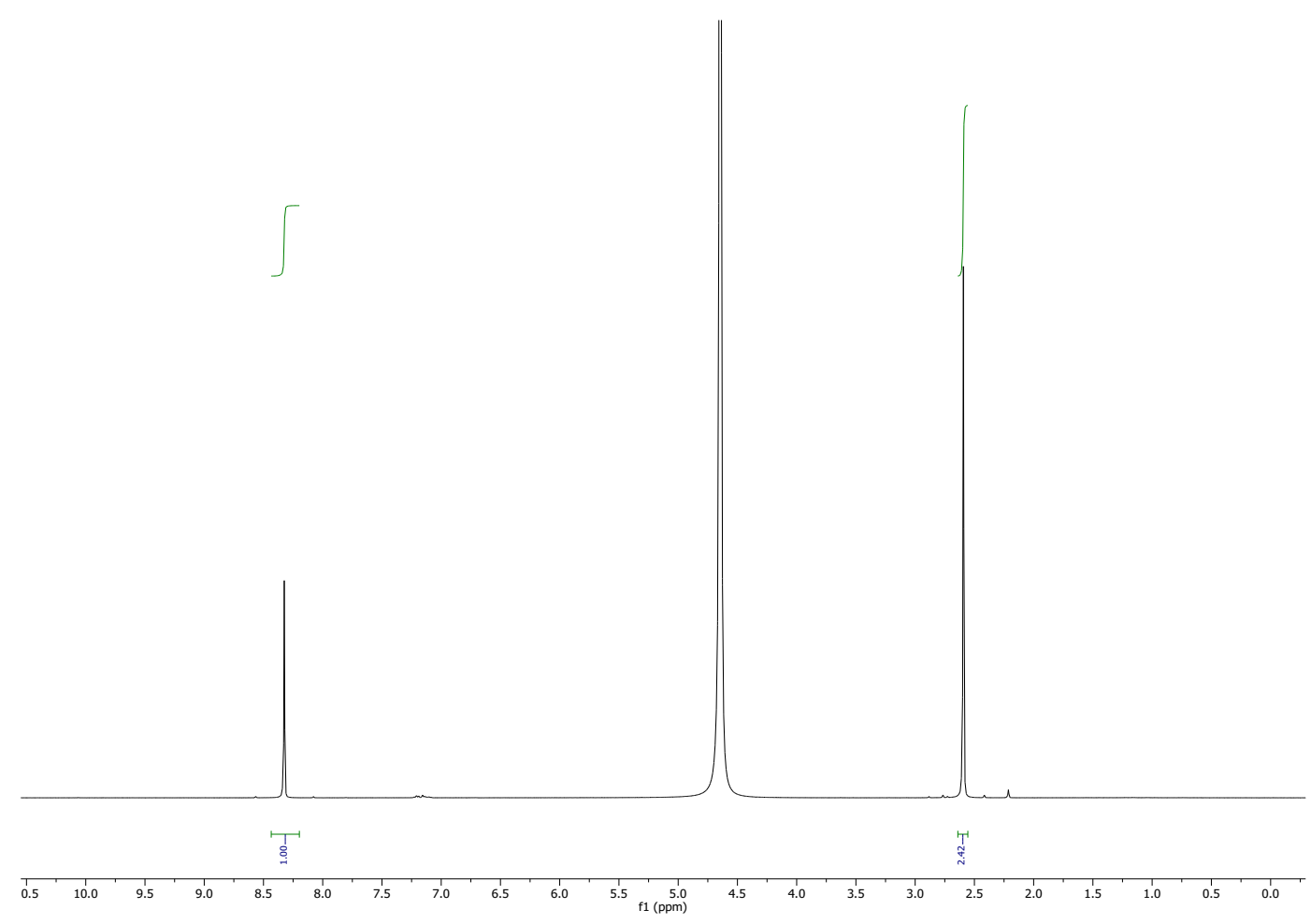

Figure S35. NMR data belonging to table S9, entry 2. 


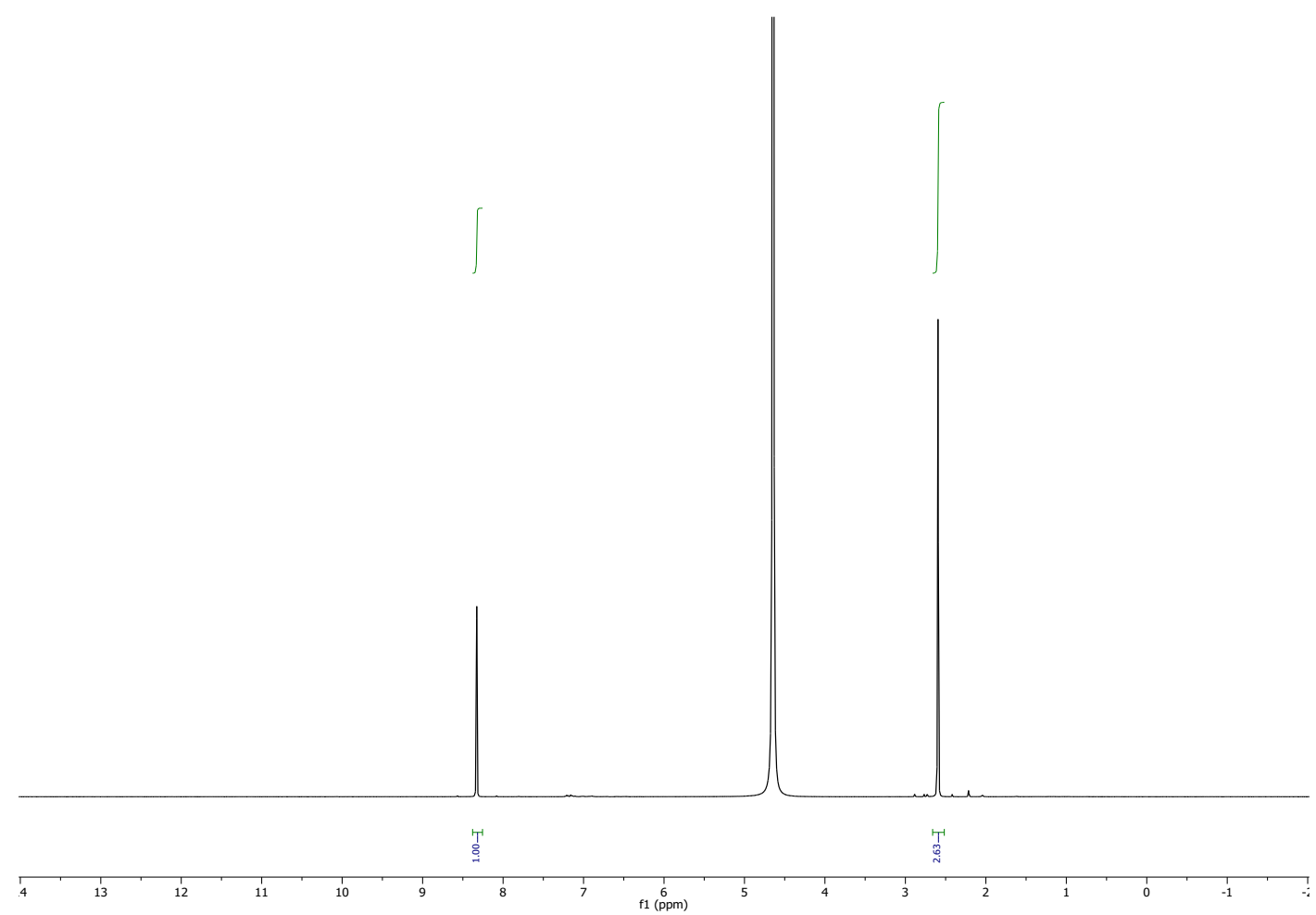

Figure S36. NMR data belonging to table S9, entry 3.

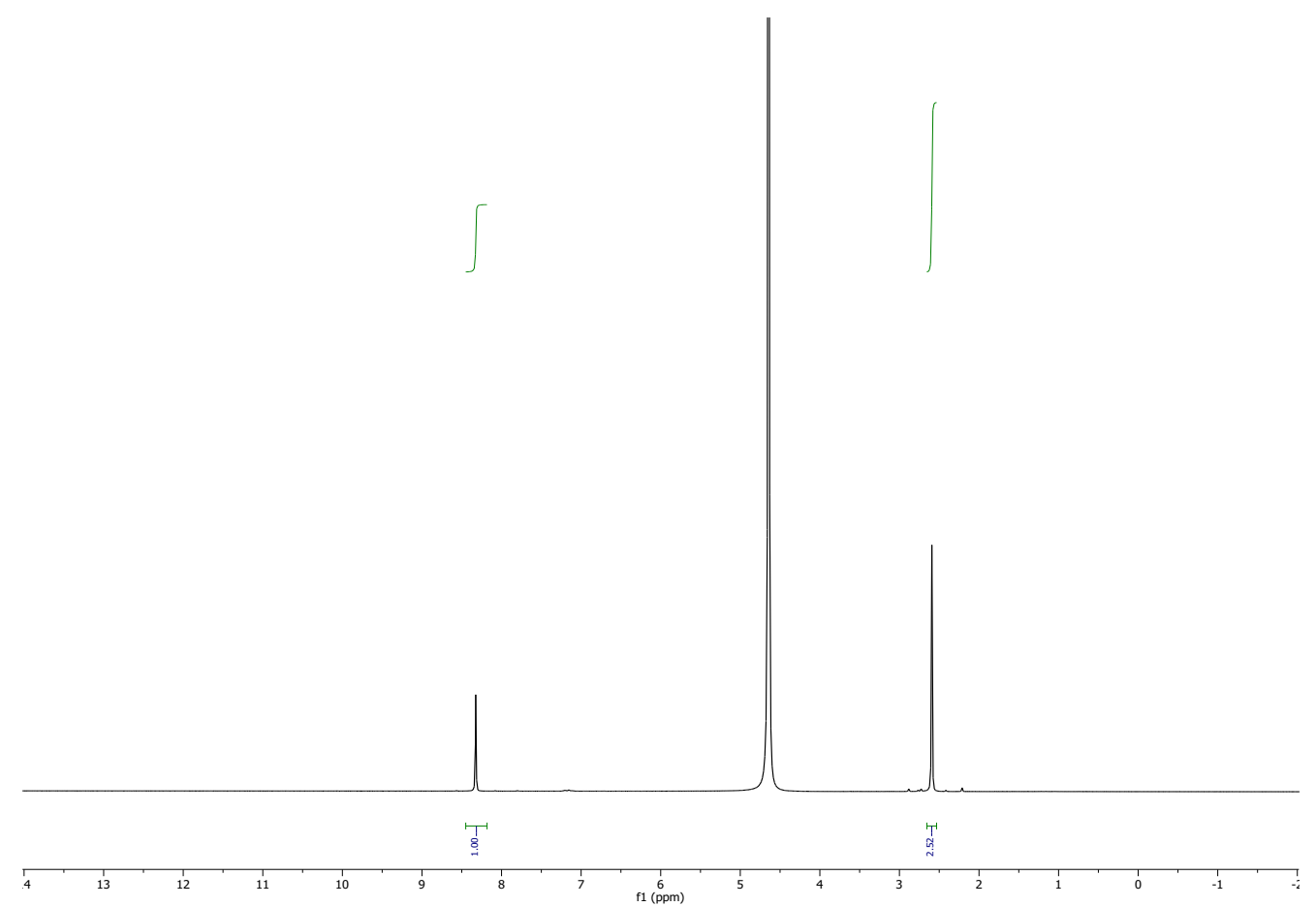

Figure S37. NMR data belonging to table S9, entry 4 . 


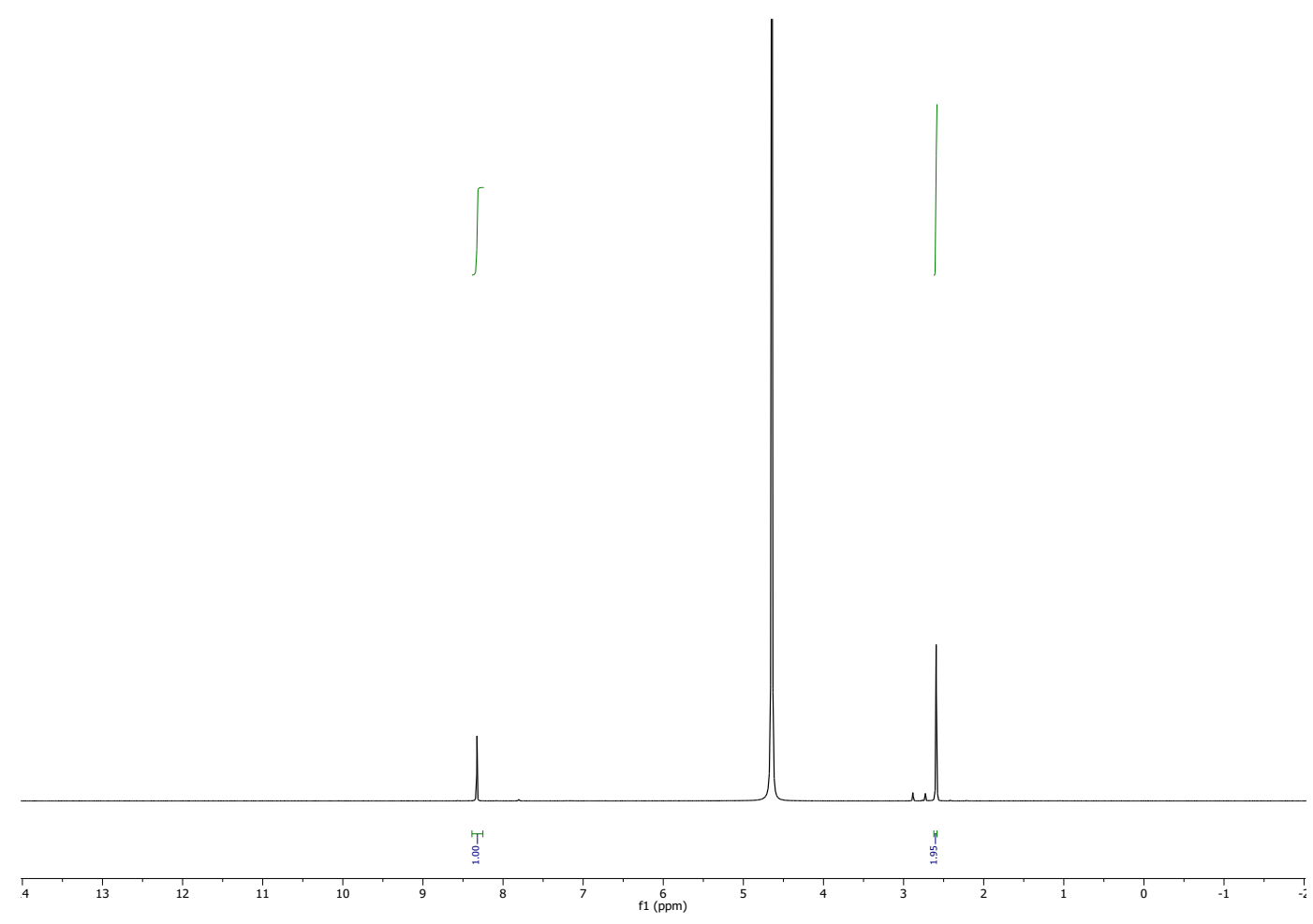

Figure S38. NMR data belonging to table S9, entry 5.

Table S9. ${ }^{1} \mathrm{H}$ NMR peak data belonging to table 4 (main text) and Figures S34-S38.

\begin{tabular}{|l|l|}
\hline Entry & Ratio of DMSO to formate integral \\
\hline 1 & 3.03 \\
\hline 2 & 2.42 \\
\hline 3 & 2.63 \\
\hline 4 & 2.52 \\
\hline 5 & 1.95 \\
\hline
\end{tabular}

\section{References:}

i. Gnanaprakasam, B.; Zhang, J.; Milstein, D. Angew. Chem. Int. Ed., 2010, 49, 1468. 